\title{
LISA: Observing Universe with Gravitational Waves from space
}

\section{Antoine Petiteau}

Antoine Petiteau, "LISA: Observing Universe with Gravitational Waves from space," Proc. SPIE 11852, International Conference on Space Optics - ICSO 2020, 1185205 (11 June 2021); doi: 10.1117/12.2599145

SPIE Event: International Conference on Space Optics - ICSO 2021, 2021, Online SPIE. Only 


\section{International Conference on Space Optics-ICSO 2020}

Virtual Conference

30 March-2 April 2021

Edited by Bruno Cugny, Zoran Sodnik, and Nikos Karafolas
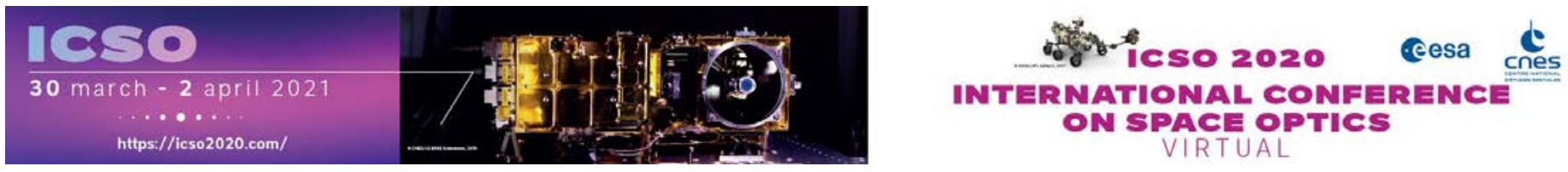

\section{LISA: Observing Universe with Gravitational Waves from space}

\section{Cesa isso procedings denes}




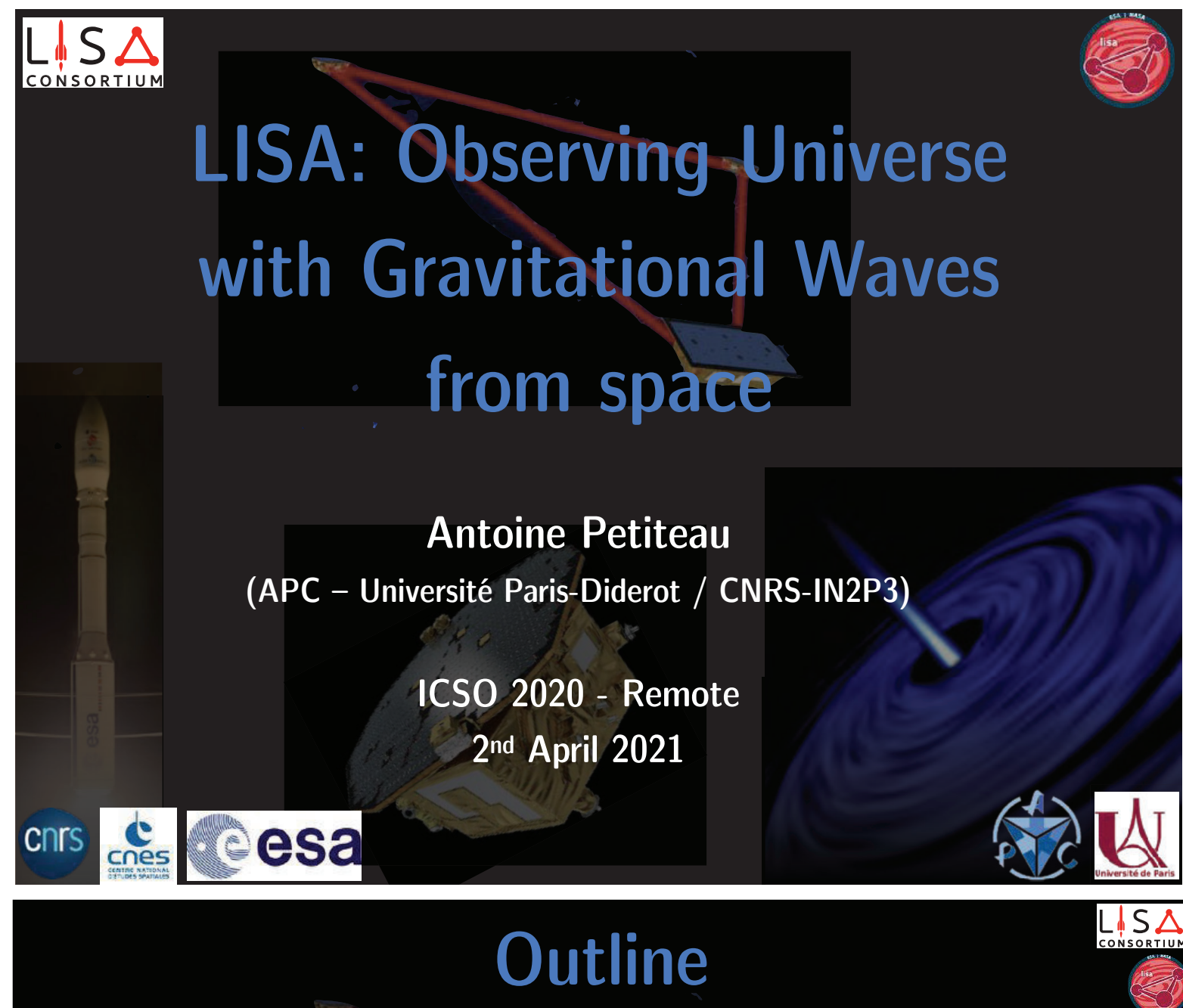

- Introduction to gravitational waves

- Gravitational wave sources

- Gravitational waves observatories

- LISA mission

- LISAPathfinder

- LISA status

- LISA scientific performances

$\checkmark$ Conclusion 


\section{Outline}

- Introduction to gravitational waves

- Gravitational wave sources

- Gravitational waves observatories

- LISA mission

- LISAPathfinder

- LISA status

- LISA scientific performances

$\checkmark$ Conclusion

\section{Some history ...}

- Albert Einstein (1905/1916) :

there is no gravity force...

- Mass deforms geometry of space-time.

- Bodies are moving in a curve space.

- Gravitational information propagates at the speed of light.

- Dissipation of energy through deformation of space-time $=>$ gravitational waves

$$
G_{\mu \nu}=\frac{8 \pi G}{c^{4}} T_{\mu \nu}
$$

geometrical distribution

deformation of energy
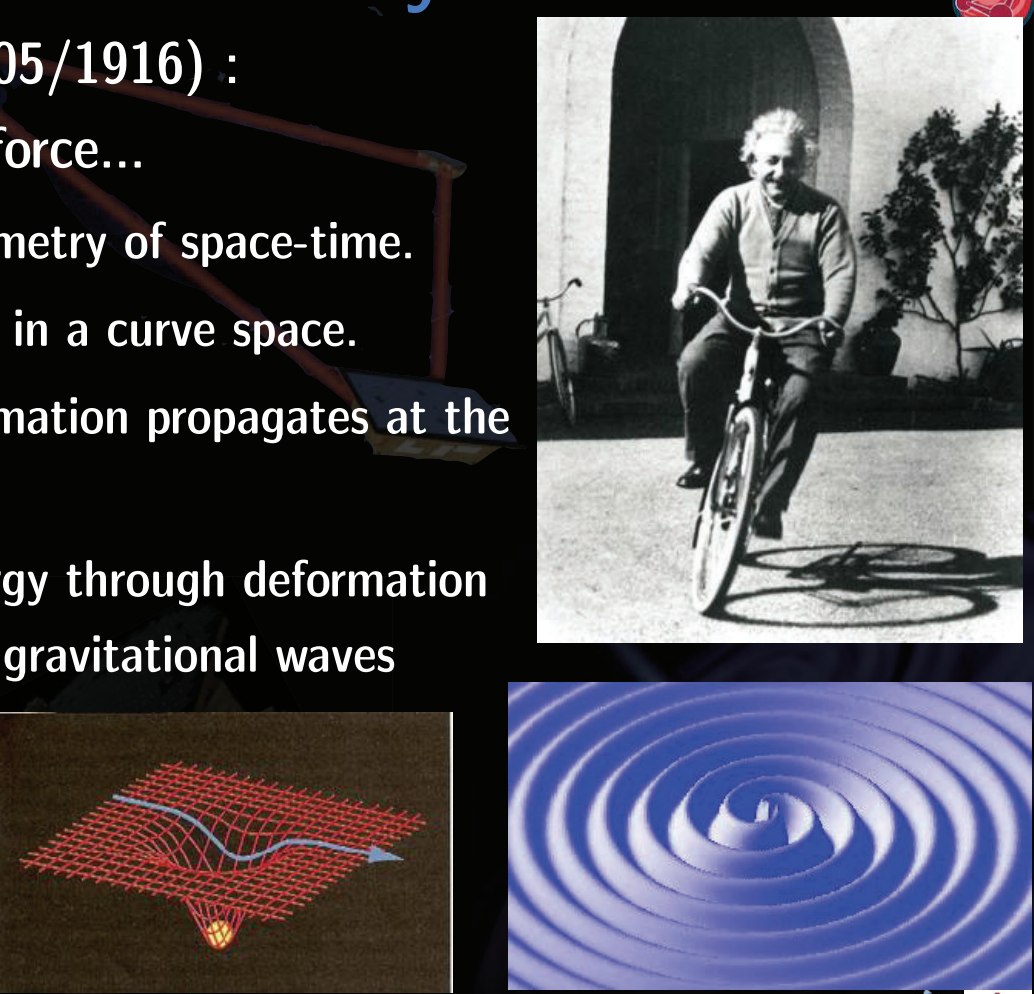

LISA - A. Petiteau - ICSO 2020 - 2nd April 2021 


\section{Emission of GWs}

- A gravitational wave is created during the non-spherical acceleration of one or several massive objects (variation of quadrupolar moment) :

- emission: asymetric collapse, bodies in orbits or coalescing, ...

- no emission: isolated, spherical body possibly in rotation

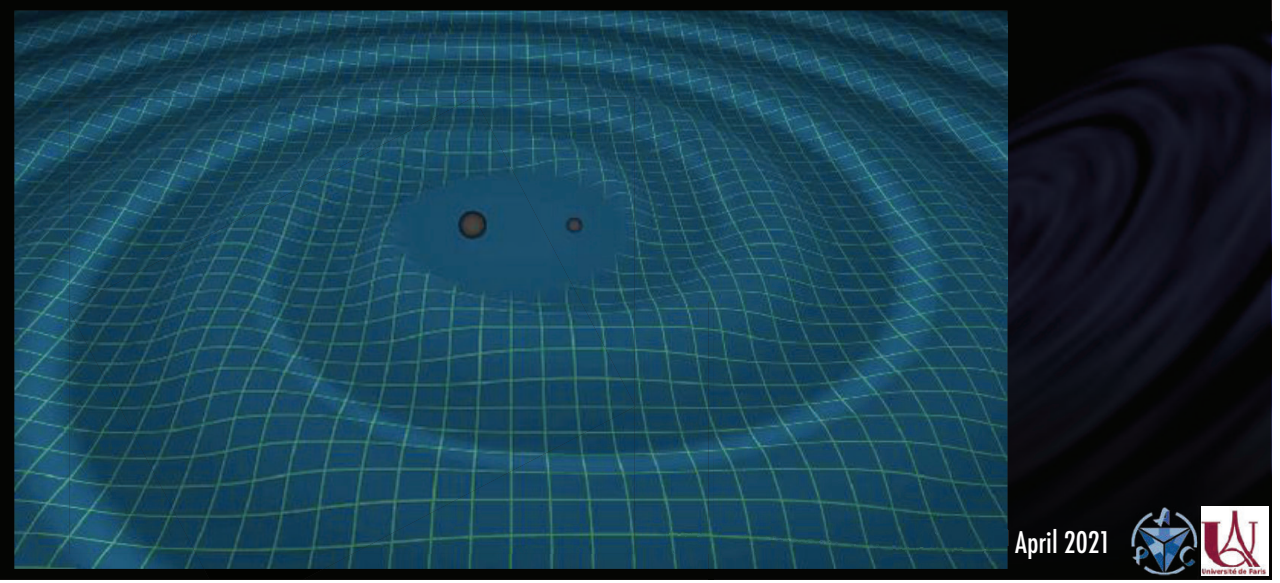

\section{Effects of GWs}

Modification of distance between 2 objects:

- Elastic deformation proportional to the distance between the 2 obj.,

- Transverse deformation: perpendicular to the direction of propagation (different from ripples on water !),

- Two components of polarisation : $\mathbf{h}_{+}$and $\mathbf{h}_{\mathbf{x}}$

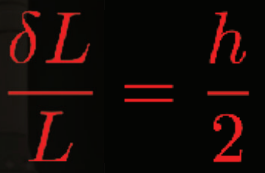

deformation wave amplitude

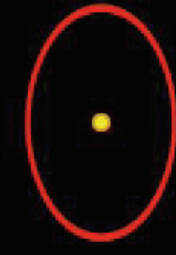

+ polarization

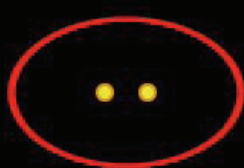

LISA - left polarization

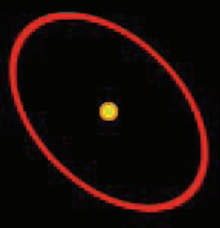

$\times$ polarization

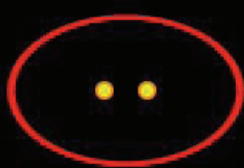

right polarization 


\section{Waveform for equal mass binaries}

- Computing waveform for Gravitational Wave:

- inspiral phase: analytic (Post-Newtonian)

- merger: numerical relativity

- ringdown (oscillation of the resulting BH): semi-analytic
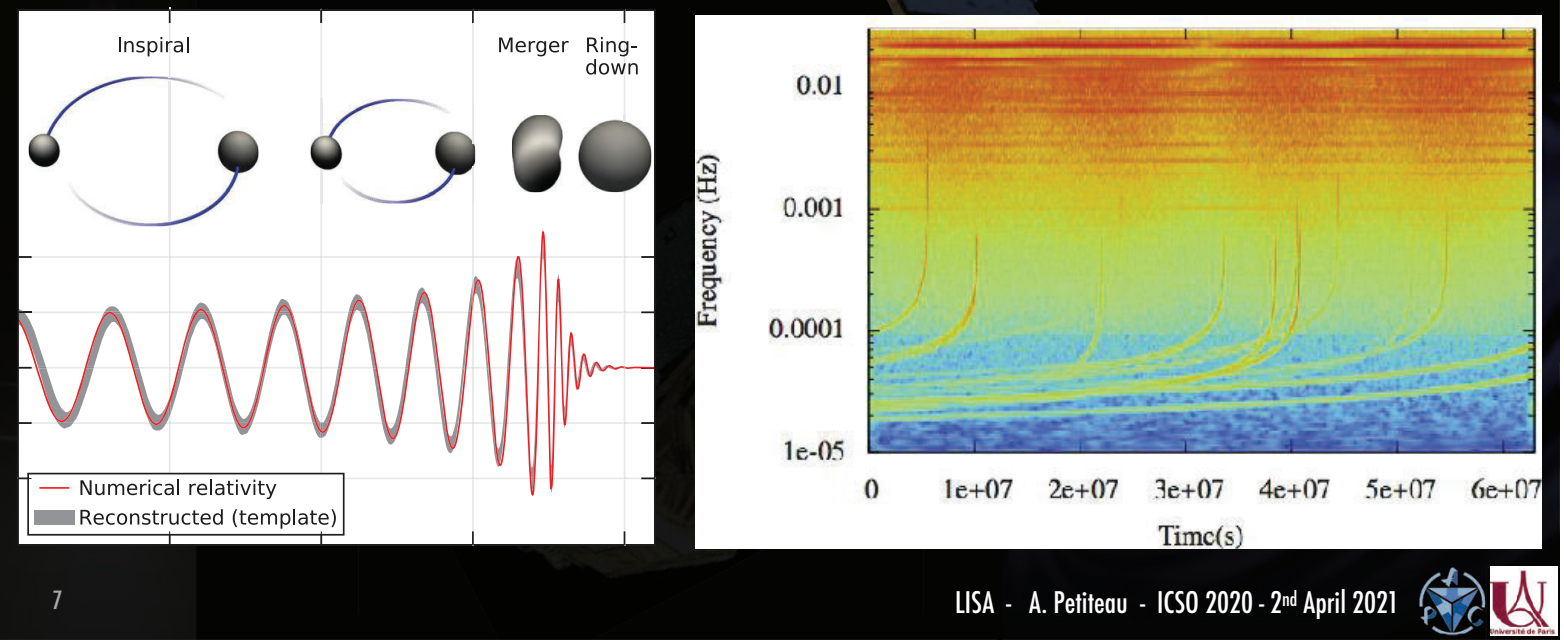

ISA - A. Petiteau - ICSO 2020 - 2nd April 2021

\section{Example: black hole binaries}

- Black hole binaries are very "simple" systems described by only 17 parameters:

- Intrinsic parameters:

- masses $\left(m_{1}\right.$ et $\left.m_{2}\right)$

- frequency and phase

- spins (amplitude and direction)

- eccentricity (value et position of periastron)

- Extrinsic parameters: position source with respect to observer

- sky position (2 angles)

- orientation (2 angles)

- time reference (time at coalescence) 


\section{Outline}

- Introduction to gravitational waves

- Gravitational wave sources

- Gravitational waves observatories

- LISA mission

- LISAPathfinder

$\checkmark$ LISA status

- LISA scientific performances

$\checkmark$ Conclusion

\section{Compact solar mass binaries}

- Large number of stars are in binary system.

- Evolution in white dwarf (WD) and neutron stars (NS).

$=>$ existence of WD-WD, NS-WD and NS-NS binaries

- Estimation for the Galaxy: 60 millions.

1 Gravitational waves:

- most part in the slow inspiral regime (quasi-monochromatic): $\mathrm{GW}$ at $\mathrm{mHz}$

- few are coalescing: GW event of few seconds at $\mathrm{f}>10 \mathrm{~Hz}$ (LIGO/Virgo)

- Several known system emitting around the $\mathrm{mHz}$

$=>$ guaranteed sources
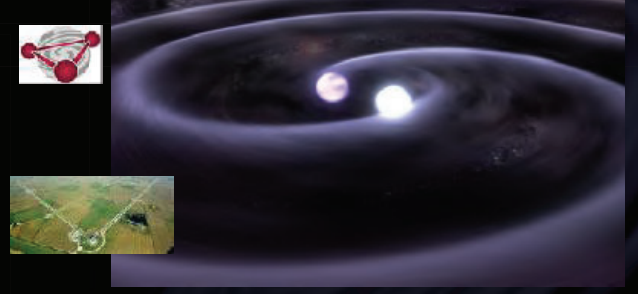

LISA - A. Petiteau - ICSO 2020 - 2nd April 2021 


\section{Black Hole Binaries}

- Binaries with 2 black holes of masses between few $\mathrm{M}_{\text {sun }}$ and $100 \mathrm{M}_{\text {Sun }}$, so called "Stellar mass BH Binaries"

- Inspiral: emission in the $\mathrm{mHz}$ band

- Merger: powerful emission around few tens $\mathrm{Hz}$

$=>$ many sources already observed

D Fast evolution: few years

from tens $\mathrm{mHz}$ to tens $\mathrm{Hz}$

$=>$ multi-observatories

observations

\section{Supermassive Black Holes}

> Observations:

- Sgr $\mathrm{A}^{*}: 4.5 \times 10^{6} \mathrm{M}$ sun at the center of the Milky Way (VLT - Gravity)

- M87: 6.5x109 Msun (picture EHT)

- Supermassive Black Hole are indirectly observed in the centre of a large number of galaxies (Active Galactic Nuclei).

- Observations of galaxy mergers $=>$

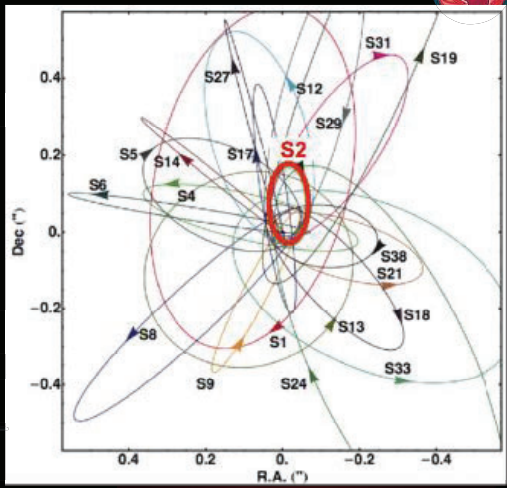

(C) Vincent, Paumard, Gourgoulhon, Perrin (2011)

$=>$ SuperMassive BH Binaries (SMBHB) should exist.
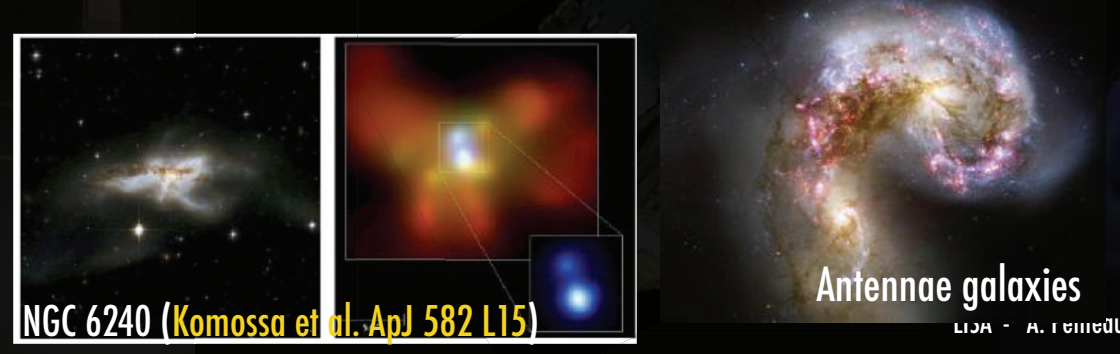

(C) EHT (2019) 


\section{MBH: Formation \& Evolution}

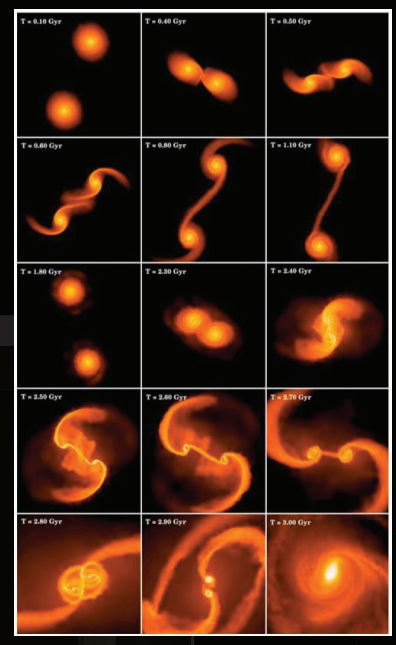

$\therefore$ Colpi \& Dotti (2009) Review, astro-ph 0906.4339

\& Talk F. Combes

* Talk J. de Freitas-Pacheco

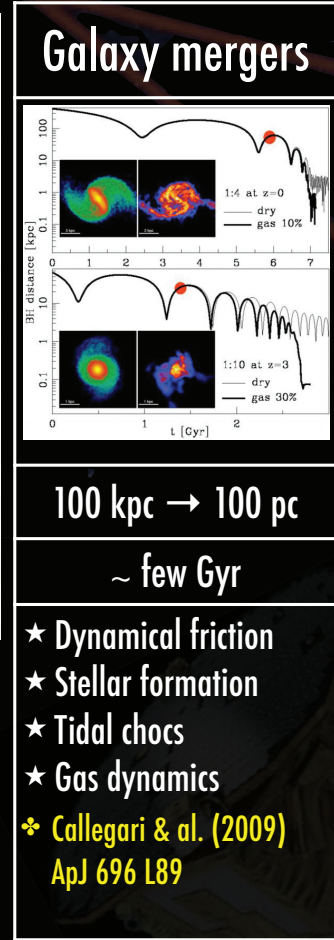

Formution

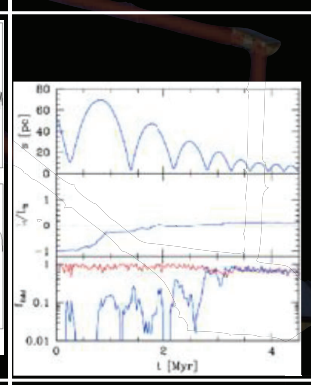

$100 \mathrm{pc} \rightarrow$ sub-parsec $\sim$ few Myr

$\star$ Gaz friction

$\star$ Circularisation

$\star$ Possible inversion of angular momentum

$\star 3$ bodies interaction

- Dotti \& al. (2009) MNRAS $396-1640$

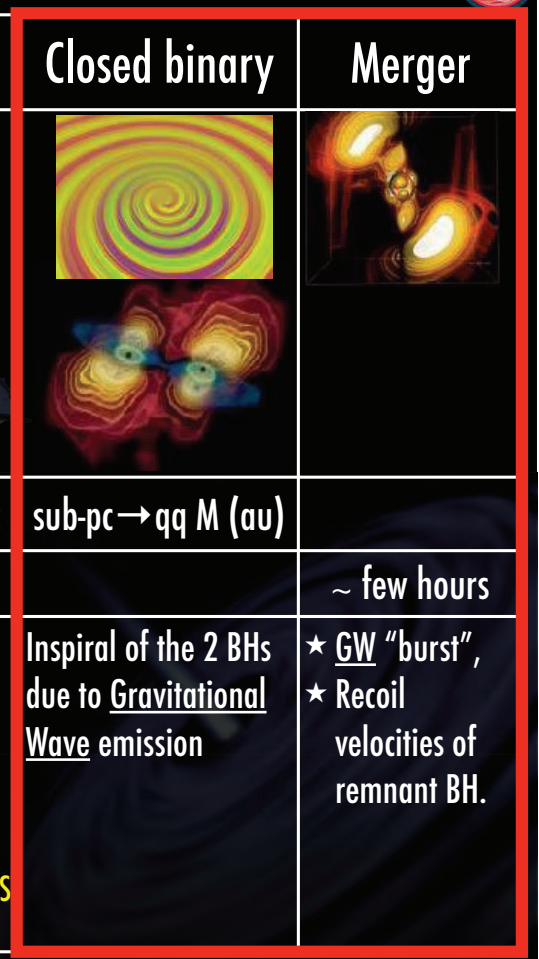

\section{Super Massive Black Hole Binaries}

- Mass $>10^{5} \mathrm{M}_{\text {Sun }}$

\ Gravitational wave:

- Inspiral: Post-Newtonian

- Merger: Numerical relativity,

- Ringdown: Oscillation of the

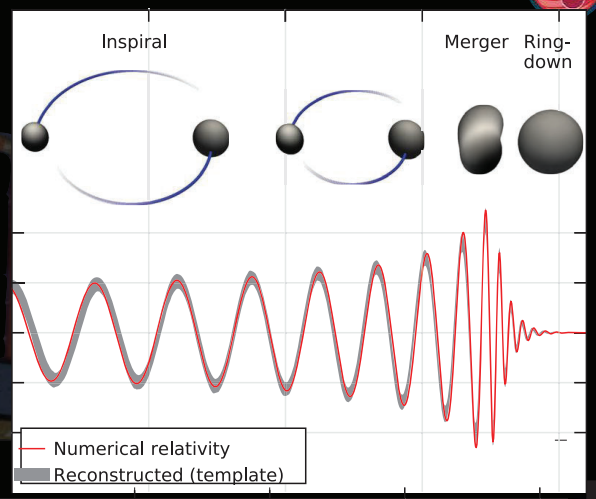
resulting $\mathrm{MBH}$.

- Inspiral at frequency $<10 \mathrm{mHz}$ (depend on the mass)
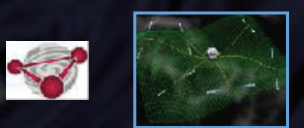

- Merger and ringdown around $\mathrm{mHz}$ 


\section{EMRIs}

- Capture of a "small" object by massive black hole $\left(10\right.$ - $\left.10^{6} \mathrm{M}_{\text {sun }}\right)$ : Extreme Mass Ratio Inspiral

- Mass ratio $>200$

- GW gives information on the geometry around the black hole.

- Test General Relativity in stong field

- Frequency : $0.1 \mathrm{mHz}$ to $0.1 \mathrm{~Hz}$

- Large number of source could be observed by space-based interferometer

\section{EMRIs}

- Small Black Hole orbiting around a SuperMassive Black Hole

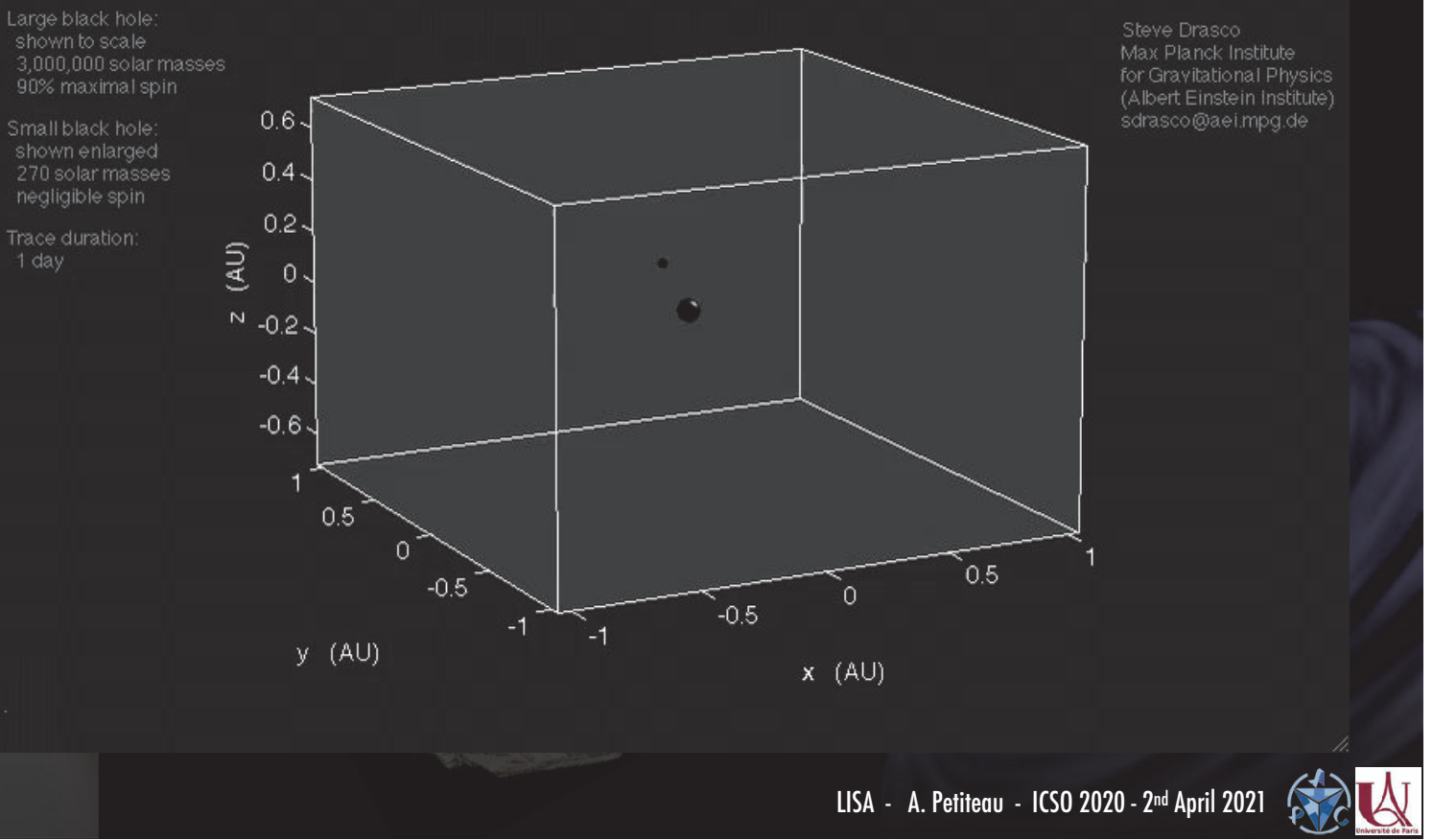




\section{Cosmological backgrounds}

- Variety of cosmological sources for stochastic background :

- First order phase transition in the very early Universe

- Cosmic strings network
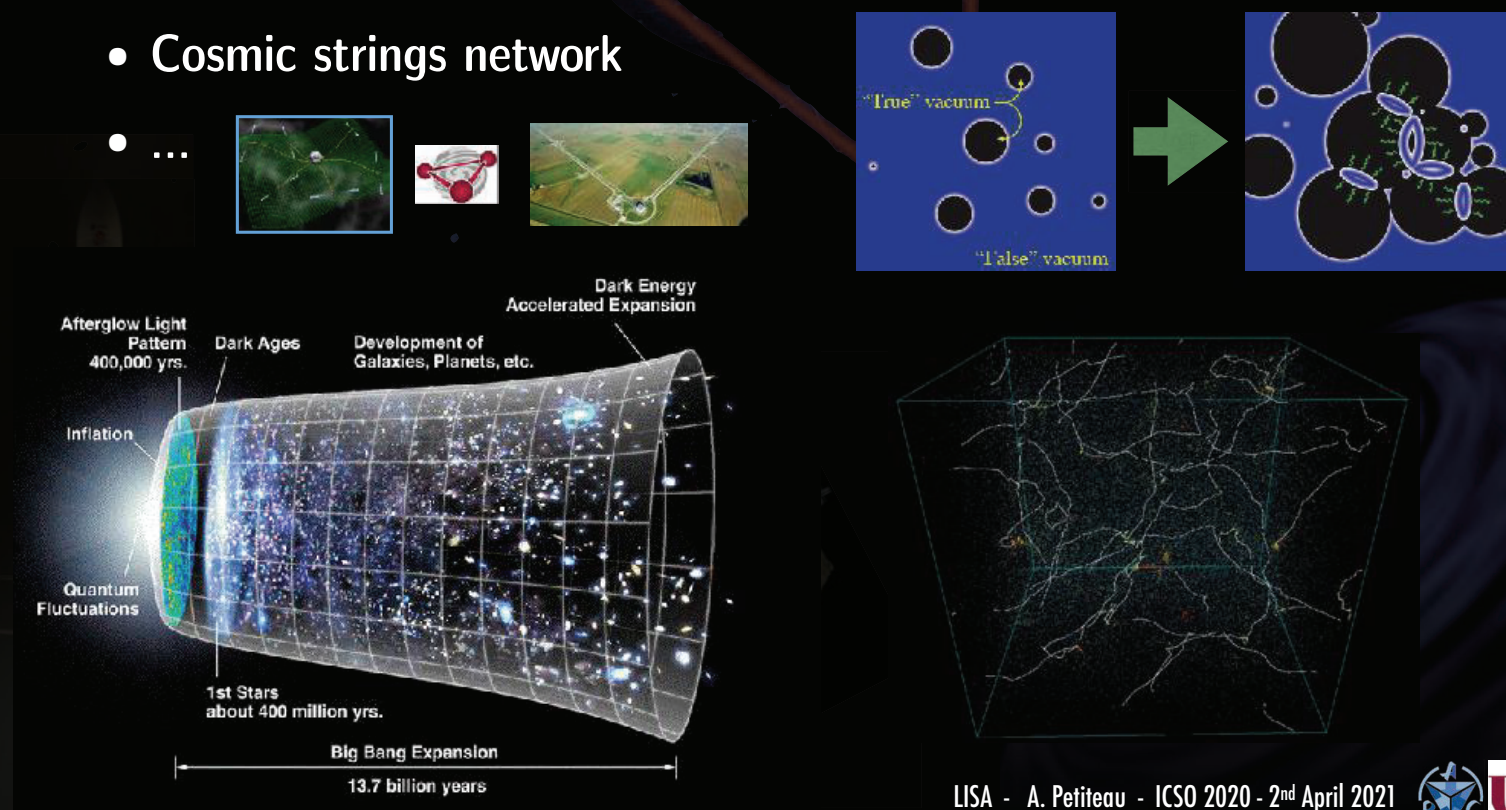

\section{Unknown sources}

- High potential of discovery ... 


\section{What can we learn?}

- The nature of gravity (testing the basis of general relativity)

- Fundamental nature of black hole: existence of horizon, ...

- Black holes as a source of energy,

- Nonlinear structure formation: seed, hierarchical assembly, accretion,

- Understanding the end of the life of massive stars,

- Dynamic of galactic nuclei,

- The very early Universe: Higgs TeV physics, topological defects, ...

- Constraining cosmological models,

$\checkmark \ldots$

\section{$=>$ New observational window on the Universe (with all the} unexpected !): looking at dark side of the Universe !

\section{9}

LISA - A. Petiteau - ICSO 2020 - 2nd April 2021

\section{GW sources over frequency band LisA}

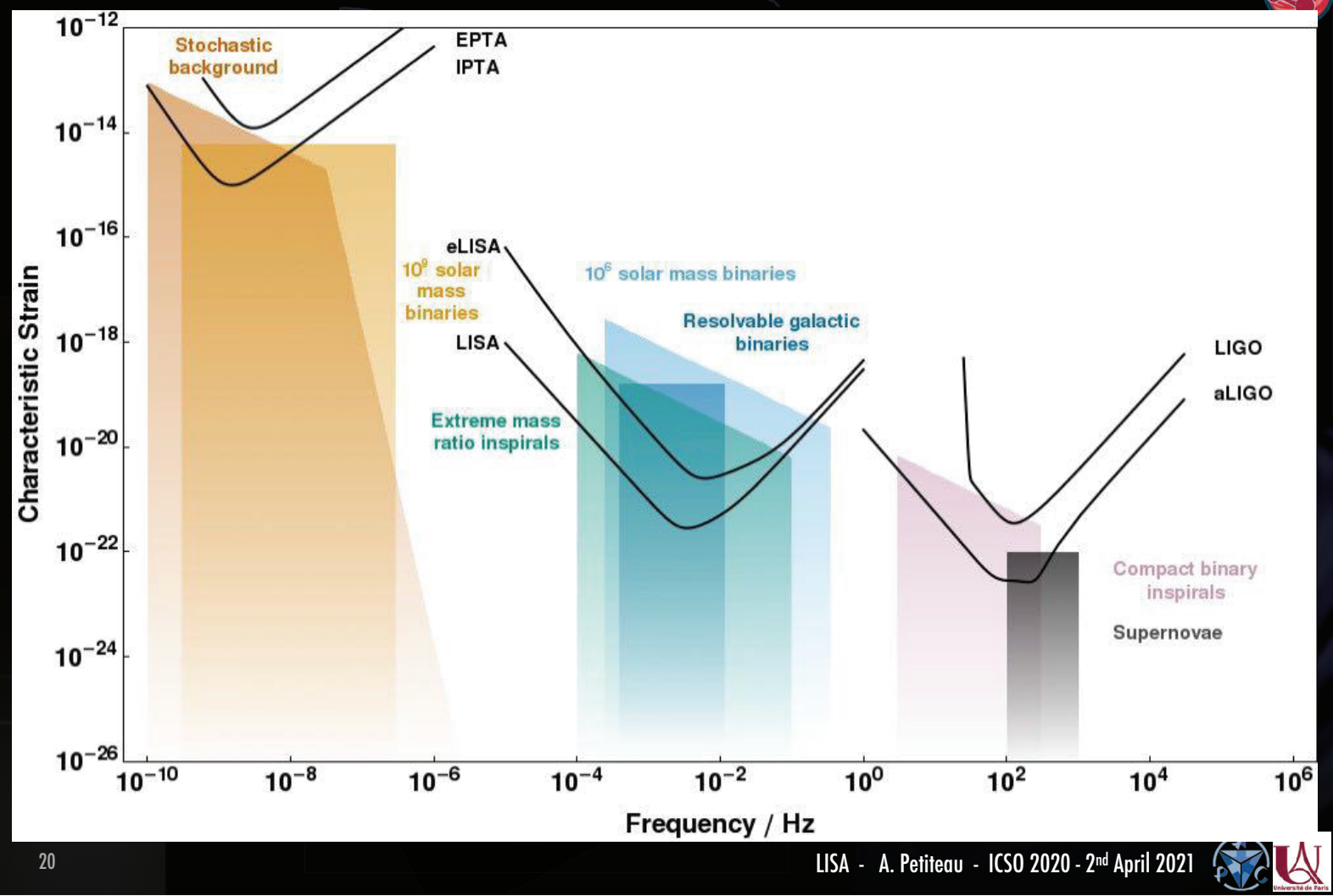




\section{Outline}

\section{$\checkmark$ Introduction to gravitational waves}

- Gravitational wave sources

- Gravitational waves observatories

- LISA mission

- LISAPathfinder

$\checkmark$ LISA status

- LISA scientific performances

> Conclusion

\section{THE GRAVITATIONAL WAVE SPECTRUM}

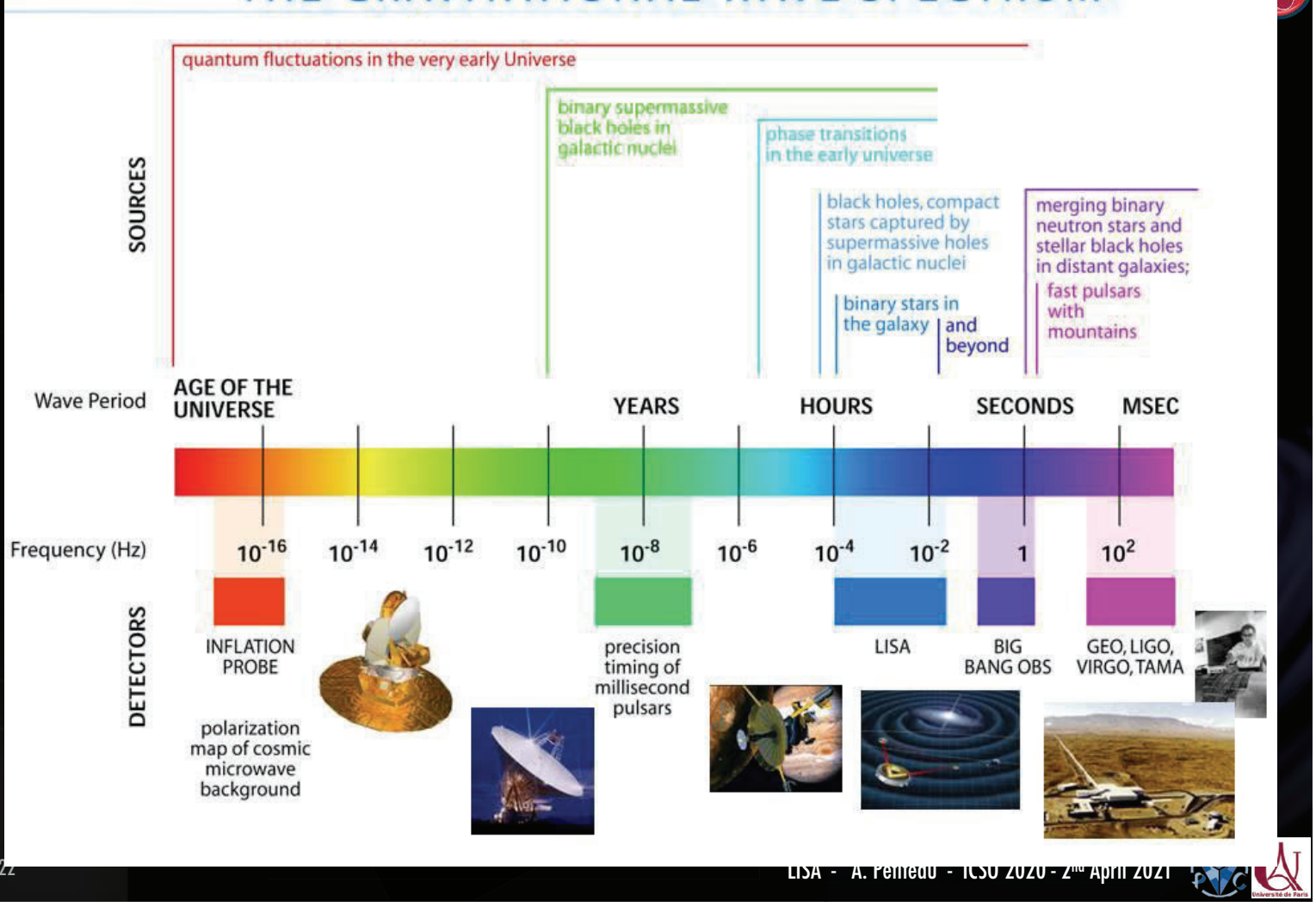




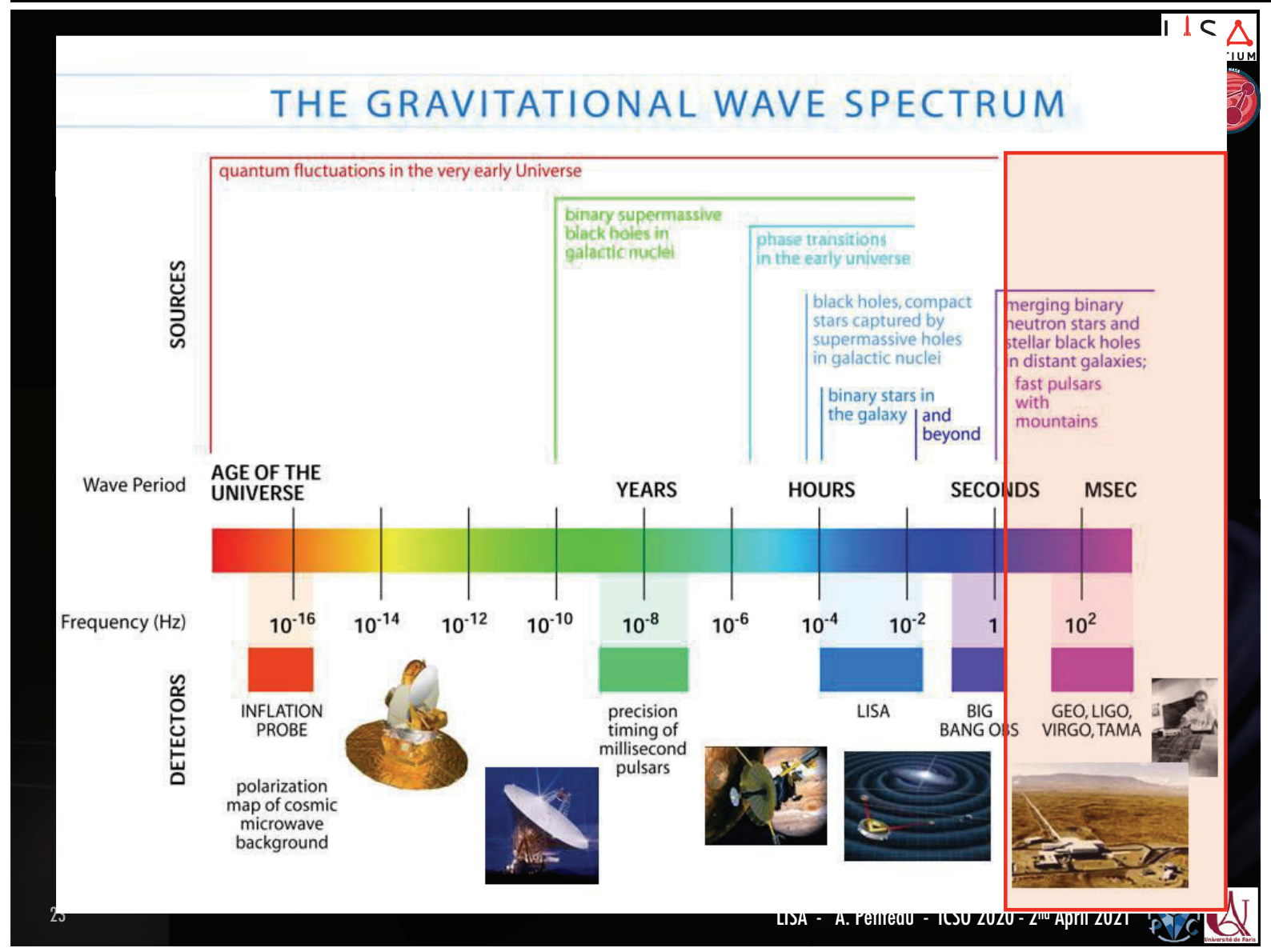

\section{Ground-based observatories

- Several interferometers

$\checkmark 50$ observed GW sources

- In the last run several events per week

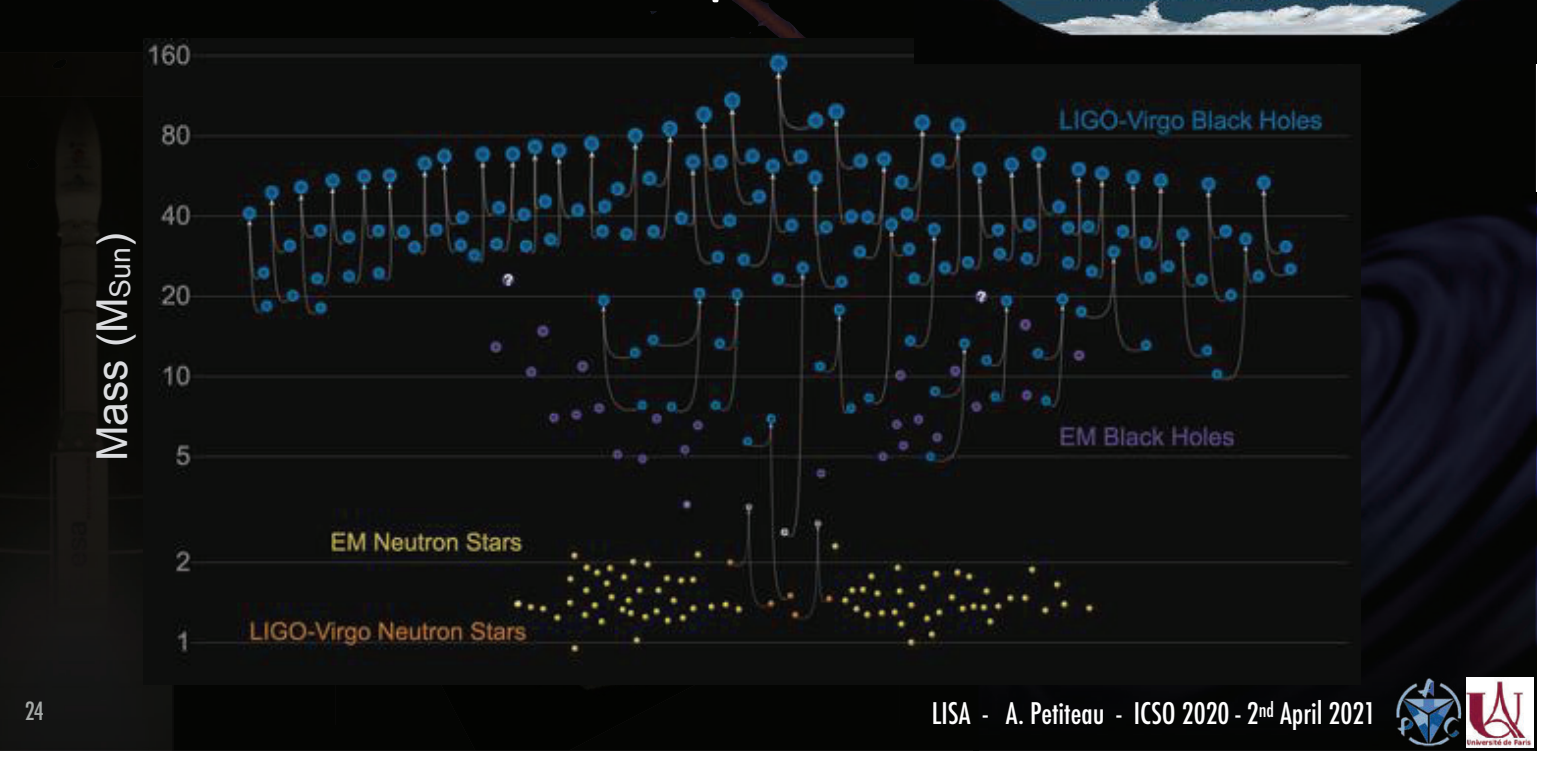

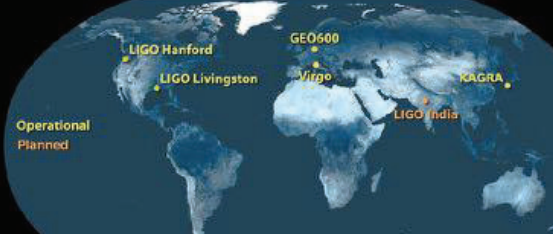

Gravitational Wave Observatories

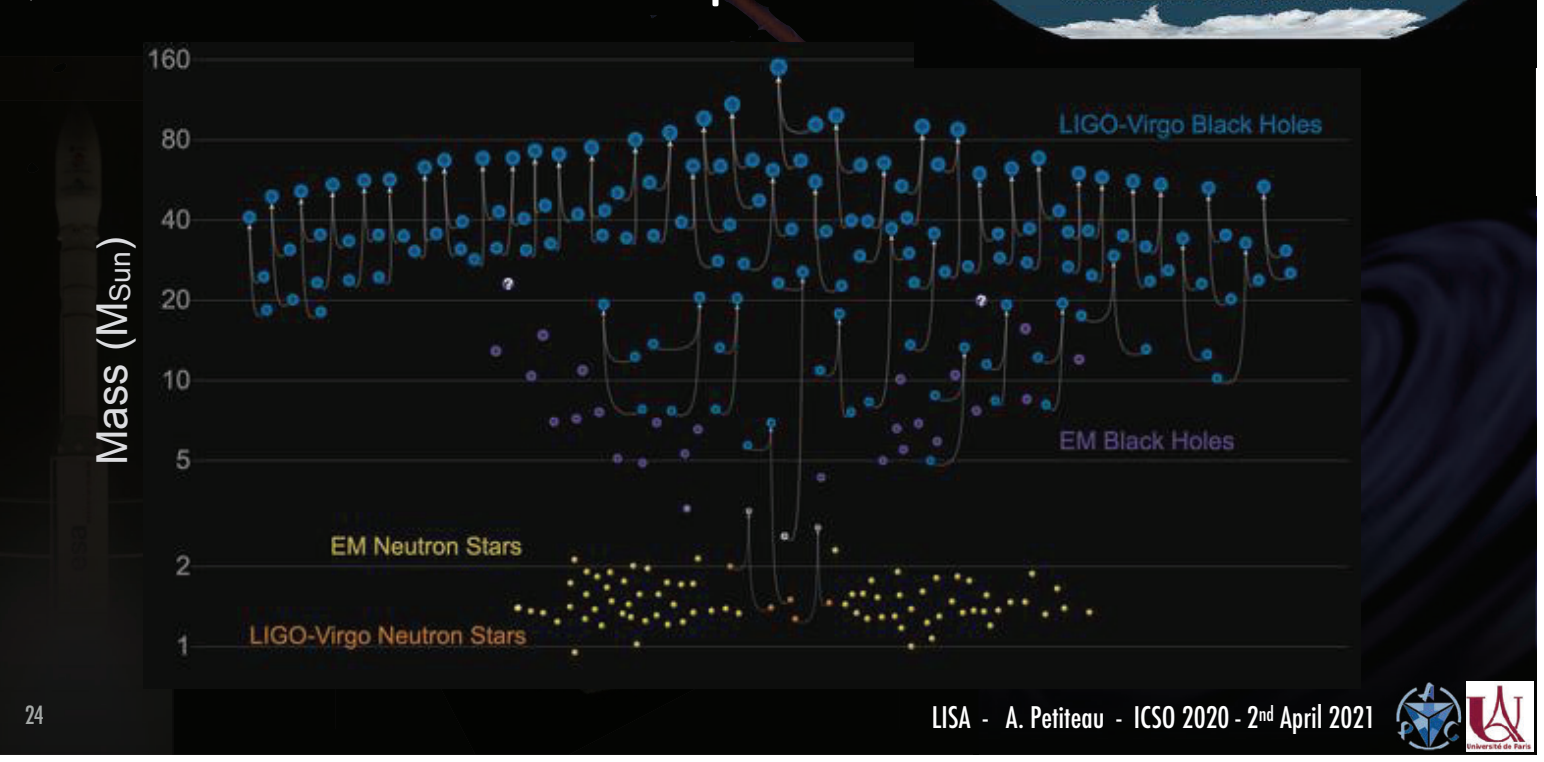




\section{Ground-based observatories}

- First obser

- BH Binar 2017 NOBEL PRIZE IN PHYSICS

- Neutron GW1708.

Binary Neutron Sta
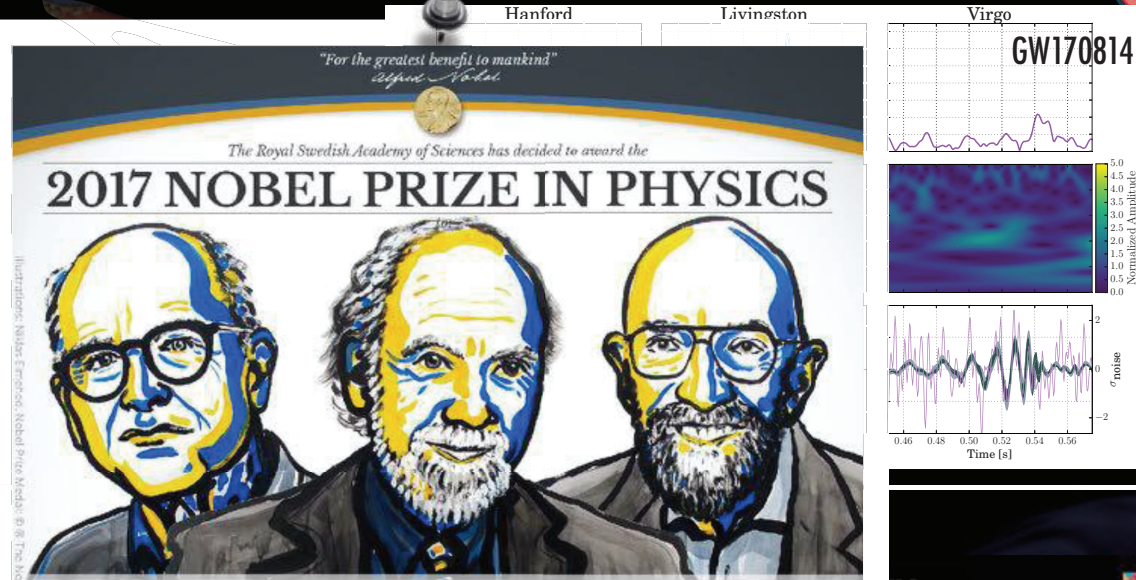

\section{Rainer Weiss Barry C. Barish Kip S. Thorne}
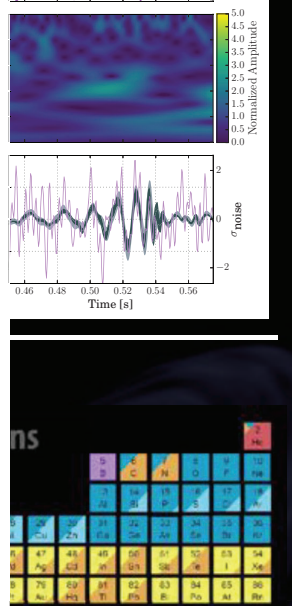

"for decisive contributions to the LIGO detector and the observation of gravitational waves" - Nobelprize.org

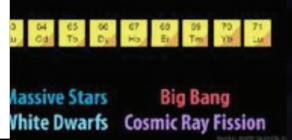

25

LISA - A. Petiteau - ICSO 2020 - 2nd April 2021

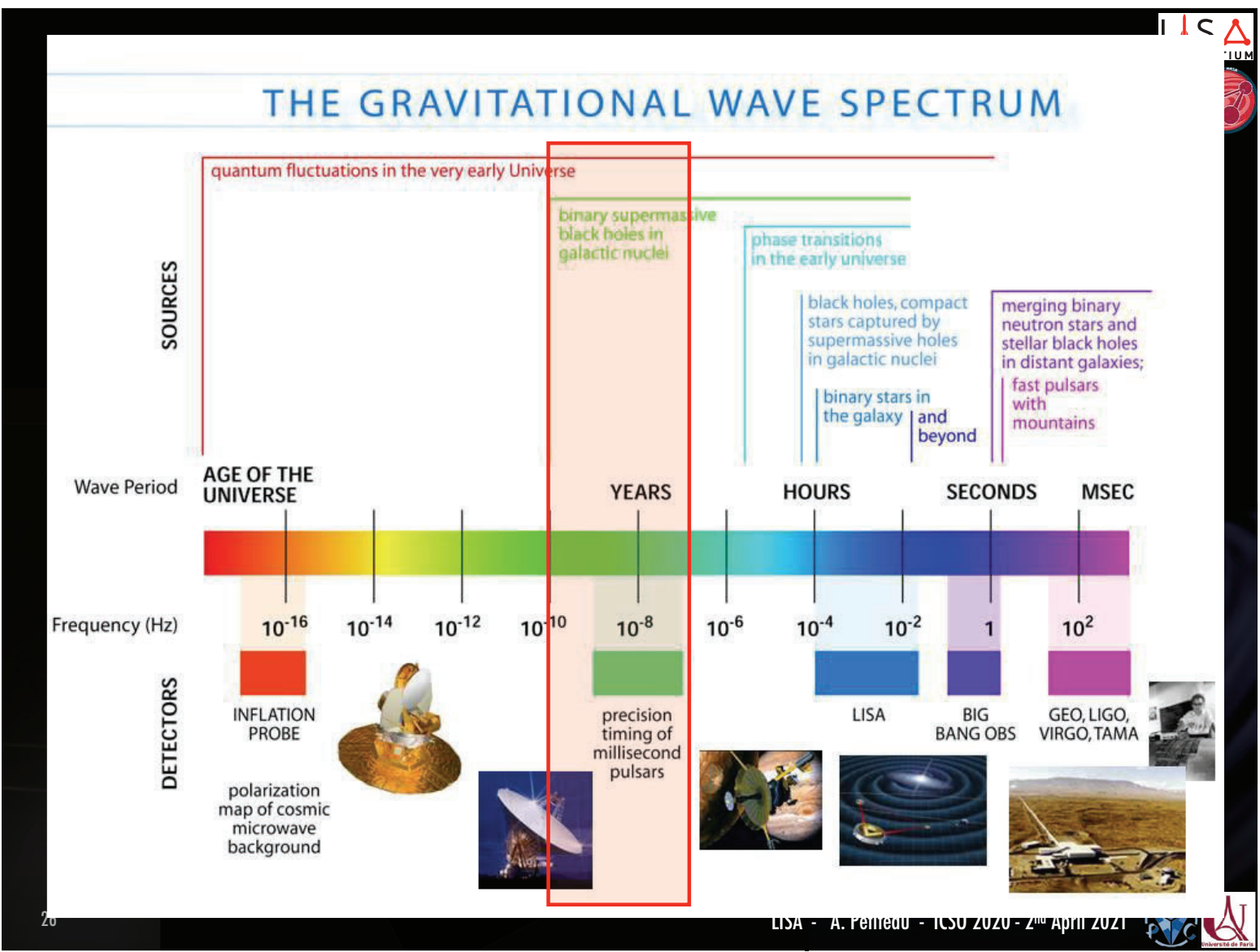




\section{Pulsar Timing Array}

- Millisecond pulsar $=$ high precision clock

- Series of extremely regular pulses are perturbed by GWs

- By timing an array of milliseconds pulsars we can detect $\mathrm{GWs}$ at $\mathrm{nHz}$

- Several collaborations (European PTA,

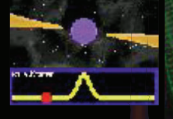
NANOGrav, PPTA, ...) grouped in IPTA

- Future: Square Kilometre Array
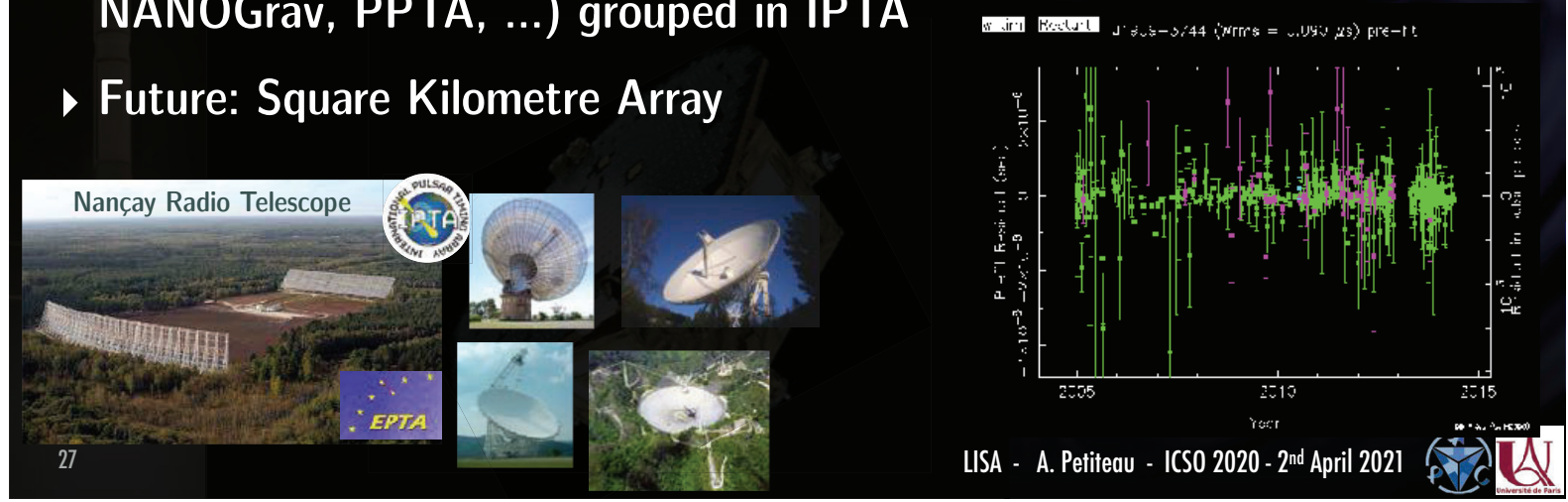

\section{Pulsar Timing Array}

D. Champion

In the nanoHz band, we are expecting:

- Close $(z<2)$ massive $\left(>10^{7} \mathrm{M}_{\text {sun }}\right)$ SuperMassive Black Hole Binaries during their inspiral phase

- Individual sources

- Stochastic background formed by the sum of a large number of SMBHB

- Cosmological background

- Recent detection of a correlated red noise in the data of NANOGrav, EPTA \& PPTA: investigation to identify if it's GW or not.
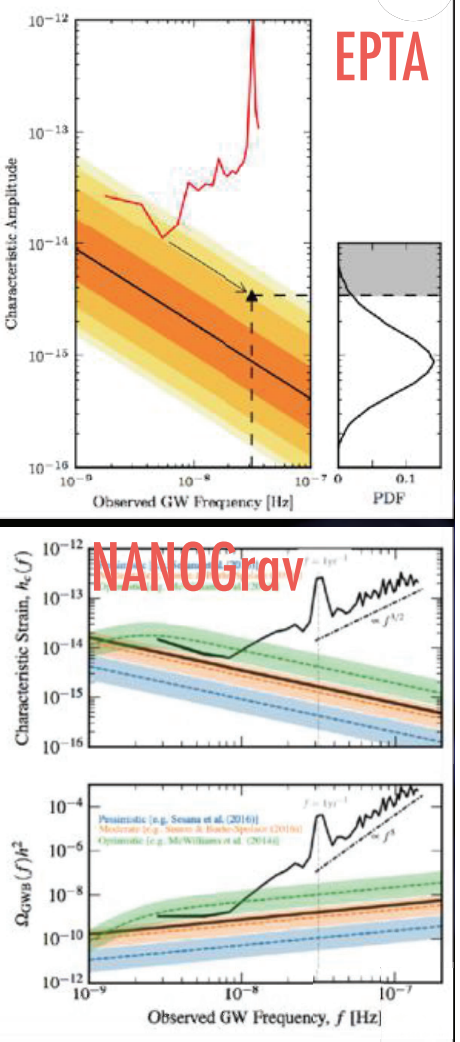


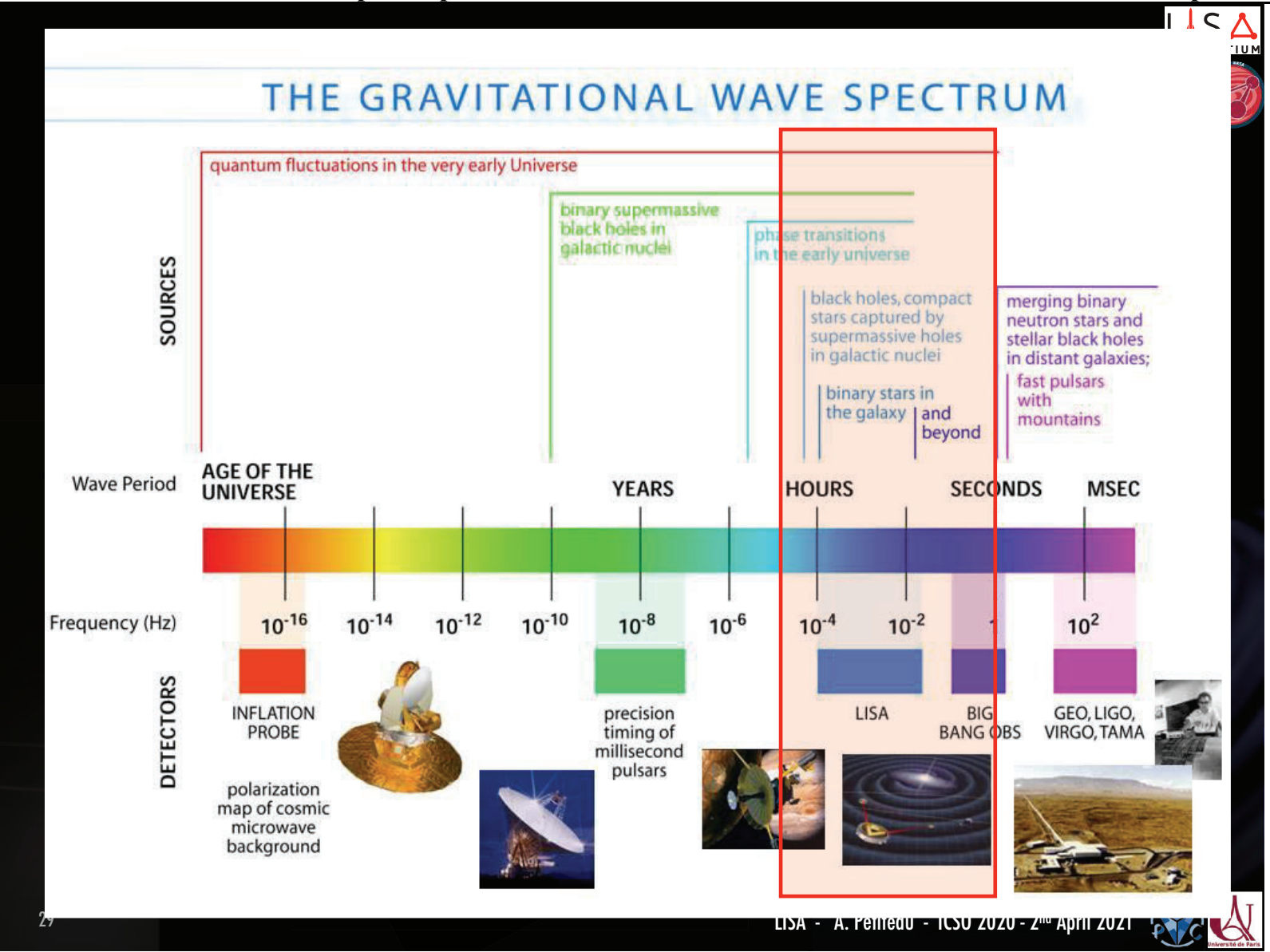

\section{Outline}

- Introduction to gravitational waves

- Gravitational wave sources

- Gravitational waves observatories

- LISA mission

- LISAPathfinder

$\checkmark$ LISA status

- LISA scientific performances

- Conclusion 


\section{LISA mission}

- Laser Interferometer Space Antenna

- 3 spacecrafts on heliocentric orbits and distant from

2.5 millions kilometers

- Goal: detect relative distance changes of 10-21: few picometers
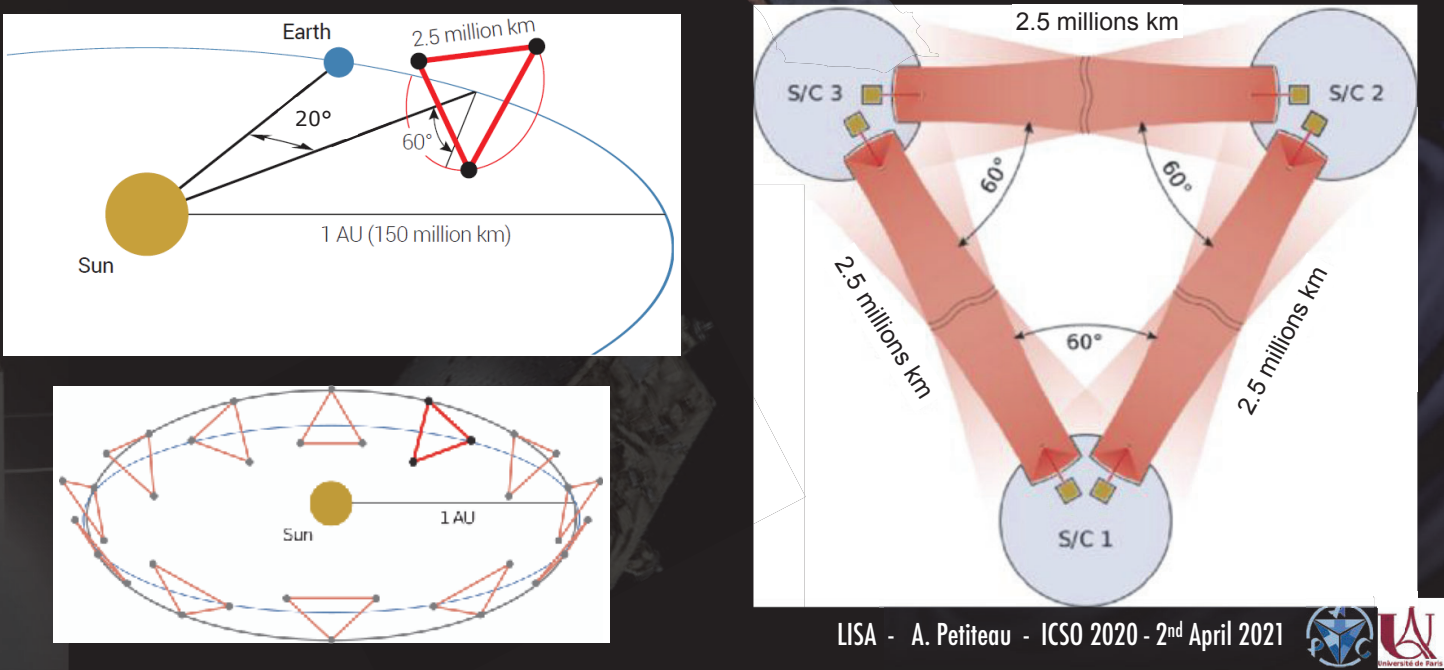

\section{LISA mission}

- Spacecraft (SC) should only be sensible to gravity:

- the spacecraft protects test-masses (TMs) from external forces and always adjusts itself on it using micro-thrusters

- Readout:

- interferometric (sensitive axis)

- capacitive sensing
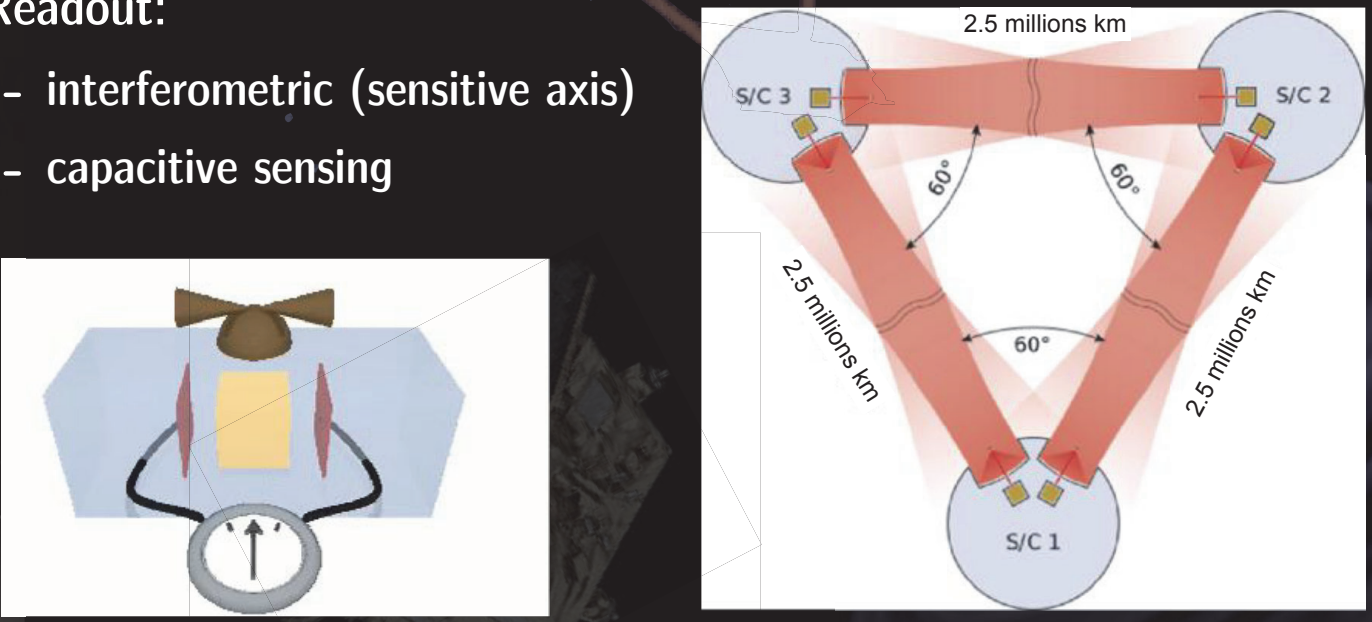

LISA - A. Petiteau - ICSO 2020 - 2nd April 2021 


\section{LISA mission}

- Several steps for an extremely precise measurements

$$
(\mathrm{TM} 2 \rightarrow \mathrm{SC} 2)+(\mathrm{SC} 2 \rightarrow \mathrm{SC} 3)+(\mathrm{SC} 3 \rightarrow \mathrm{TM} 3)
$$

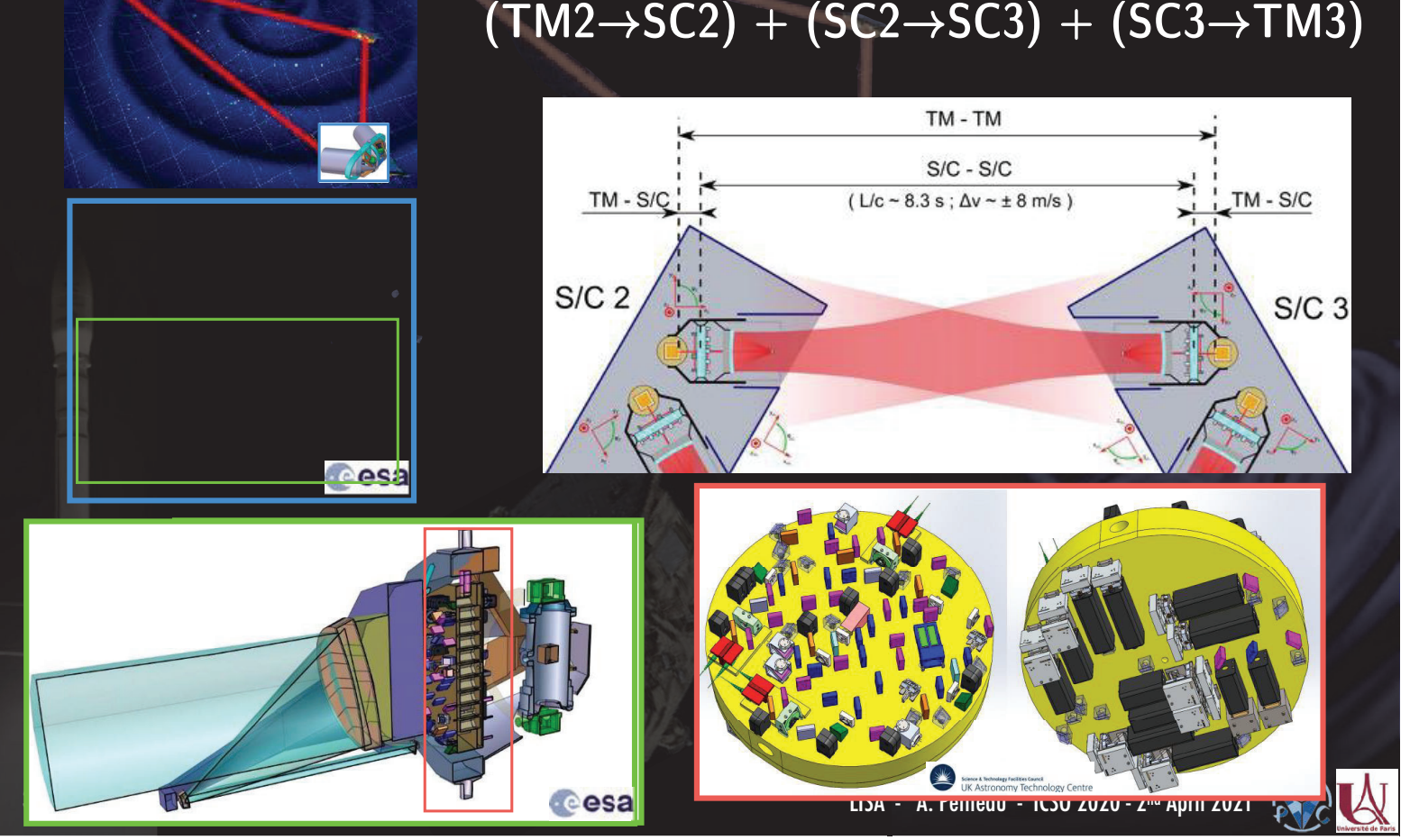

\section{LISA mission}

- Exchange of laser beams to form several interferometers

- Phasemeter measurements on each of the 6 Optical Benches:

- Distant OB vs local OB

- Test-mass vs $\mathrm{OB}$

- Reference using adjacent OB

- Transmission using sidebands

- Distance between spacecrafts

- Noises sources:

- Laser noise : $10^{-13}$ (vs $10^{-21}$ )

- Clock noise (3 clocks)

- Acceleration noise (see LPF)

- Read-out noises

- Optical path noises

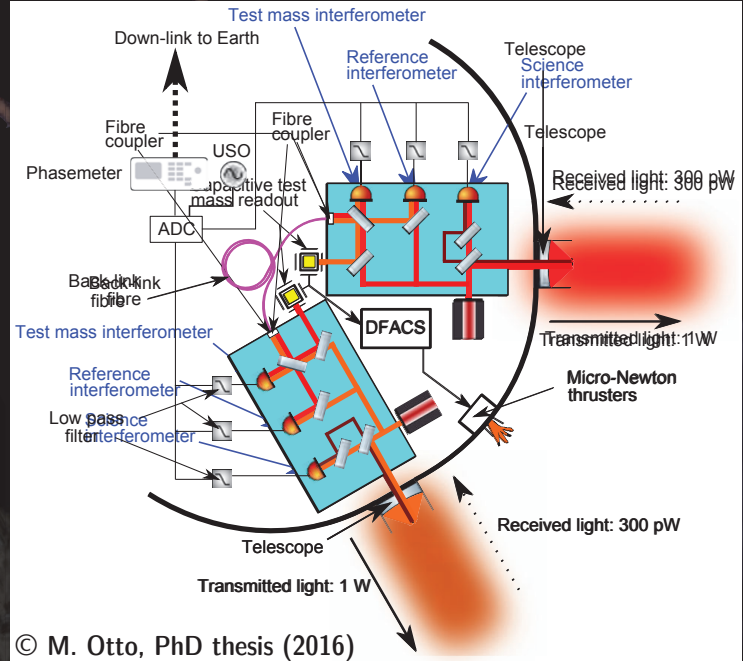

(C) M. Otto, PhD thesis (2016) 


\section{LISA mission}

\section{- Measurements via}

\section{exchange of beams:}

- Heterodyne interferometry with carrier for interspacecraft measurement $=>\mathrm{GWs}$

- Sideband for transferring amplified clock jitter $=>$ correction of additional clock jitter

- Pseudo-Random Noise $=>$ ranging (measure arm length)

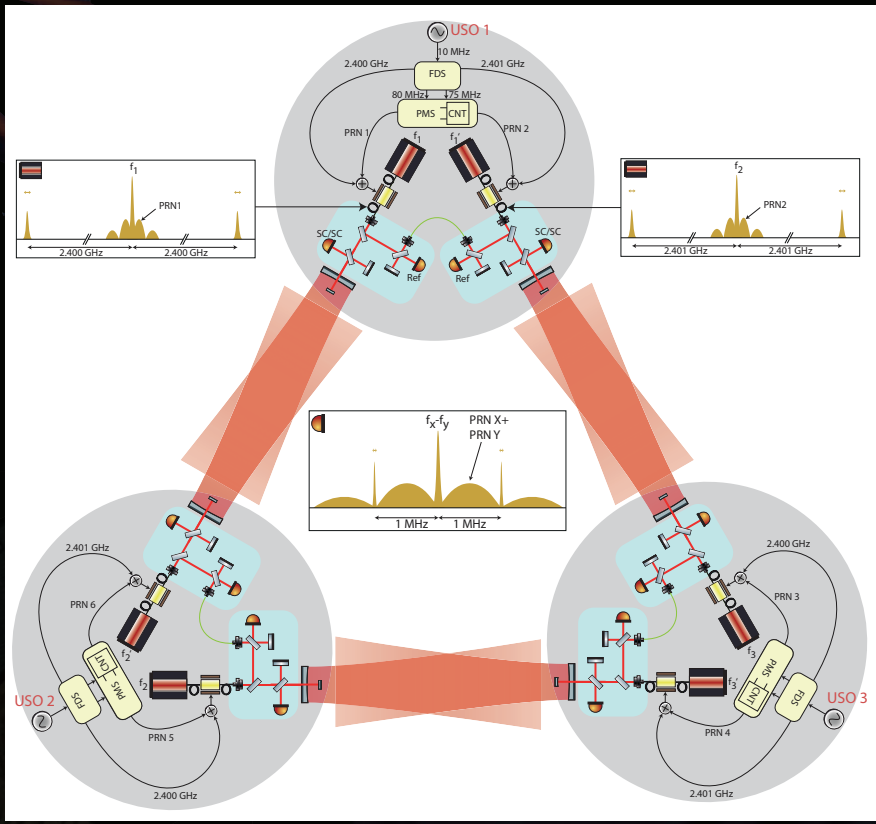

\section{LISA technology requirements}

- Free flying test mass subject to very low parasitic forces:

- Drag free control of spacecraft (non-contacting) with low noise microthruster

- Large gaps, heavy masses with caging mechanism

- High stability electrical actuation on cross degrees of freedom

- Non contacting discharging of test-masses

- High thermo-mechanical stability of spacecraft

- Gravitational field cancellation

- Precision interferometric, local ranging of test-mass and spacecraft:

- pm resolution ranging, sub-mrad alignments

○ High stability monolithic optical assemblies

- Precision million $\mathrm{km}$ spacecraft to spacecraft precision ranging:

- High accuracy laser frequency stabilization + noise suppression with TDI

○ "Tilt to length" coupling (control of alignement + ground correction)

- Low level of stray-light

- High stability telescopes

- High accuracy phase-meter and frequency distribution

- Constellation acquisition

- Precision attitude control of spacecraft 
Phasemeters (carrier, sidebands, distance)

+ Gravitational Refe-rence Sensor

'Survey' type observatory

Auxiliary channels
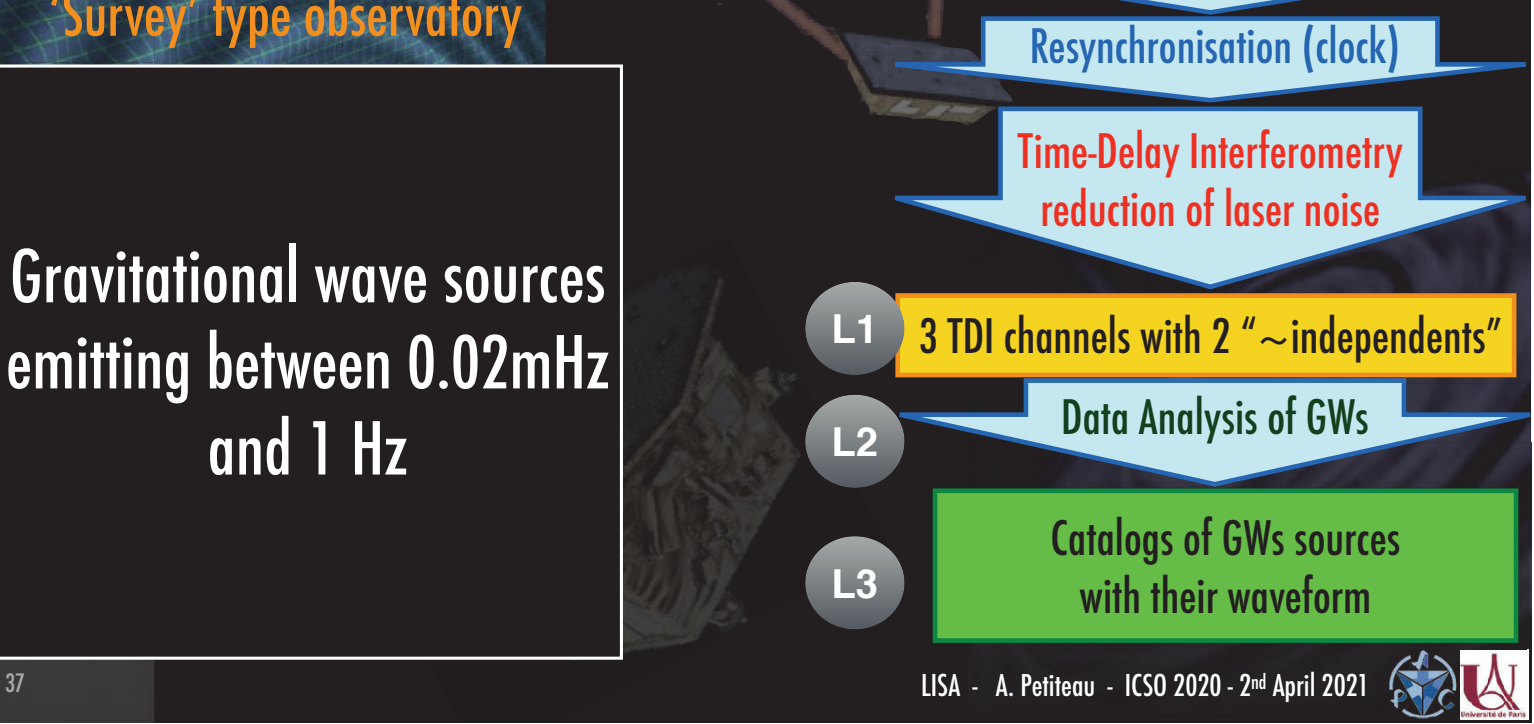

- Reducing dominating noises for producing data in which GW sources can be extracted

- Laser frequency noise reduced by $\mathbf{8}$ orders of magnitude with Time Delay Interferometry:

- Combination of delayed measurements reconstructing virtually several interferometers

$$
\begin{aligned}
X_{2}=\eta_{1^{\prime}}+\mathcal{D}_{2^{\prime}} \eta_{3}+\mathcal{D}_{2^{\prime} 2} \eta_{1}-\mathcal{D}_{2^{\prime} 23} \eta_{2^{\prime}}+\mathcal{D}_{2^{\prime} 233^{\prime}} \eta_{1} \\
+\mathcal{D}_{2^{\prime} 233^{\prime} 3} \eta_{2^{\prime}}+\mathcal{D}_{2^{\prime} 233^{\prime} 33^{\prime}} \eta_{1^{\prime}}+\mathcal{D}_{2^{\prime} 233^{\prime} 33^{\prime} 2^{\prime}} \eta_{3} \\
-\eta_{1}-\mathcal{D}_{3} \eta_{2^{\prime}}-\mathcal{D}_{33^{\prime}} \eta_{1^{\prime}}-\mathcal{D}_{33^{\prime} 2^{\prime}} \eta_{3}-\mathcal{D}_{33^{\prime} 2^{\prime} 2} \eta_{1^{\prime}} \\
-\mathcal{D}_{33^{\prime} 2^{\prime} 22^{\prime}} \eta_{3}-\mathcal{D}_{33^{\prime} 2^{\prime} 22^{\prime} 2} \eta_{1}-\mathcal{D}_{33^{\prime} 2^{\prime} 22^{\prime} 23} \eta_{2^{\prime}}
\end{aligned}
$$

$D_{i} x(t)=x\left(t-\frac{L}{c}\right)$

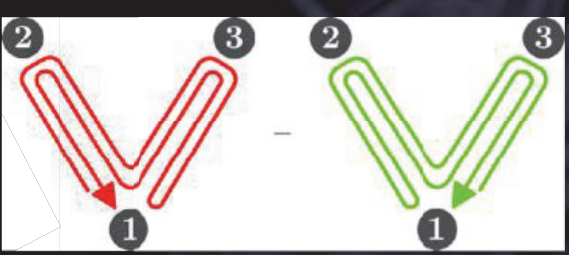

LISA - A. Petiteau - ICSO 2020 - 2nd April 2021 


\section{GW data analysis}

- Large number of overlapping sources

$=>$ challenging for data analysis

- Exploration of multiple technics

- First data of this kind + fluctuations of computational charge

(fluctuations of event rates, new calibrations, ...)

$=>$ flexible Distributed Data Processing Center

- Prototyping already started:

LISA Data Challenge,

detailed simulations

(instrument \& GW sources)

39

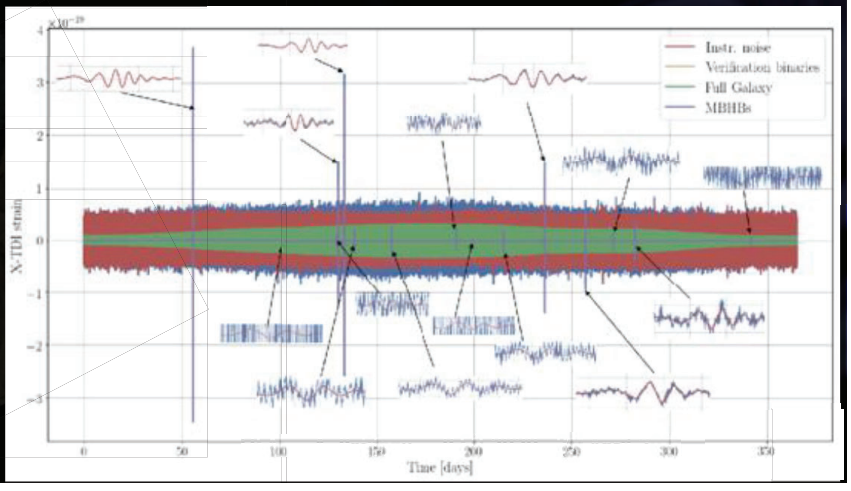

\section{Outline}

- Introduction to gravitational waves

- Gravitational wave sources

- Gravitational waves observatories

- LISA mission

- LISAPathfinder

$\checkmark$ LISA status

- LISA scientific performances

- Conclusion 


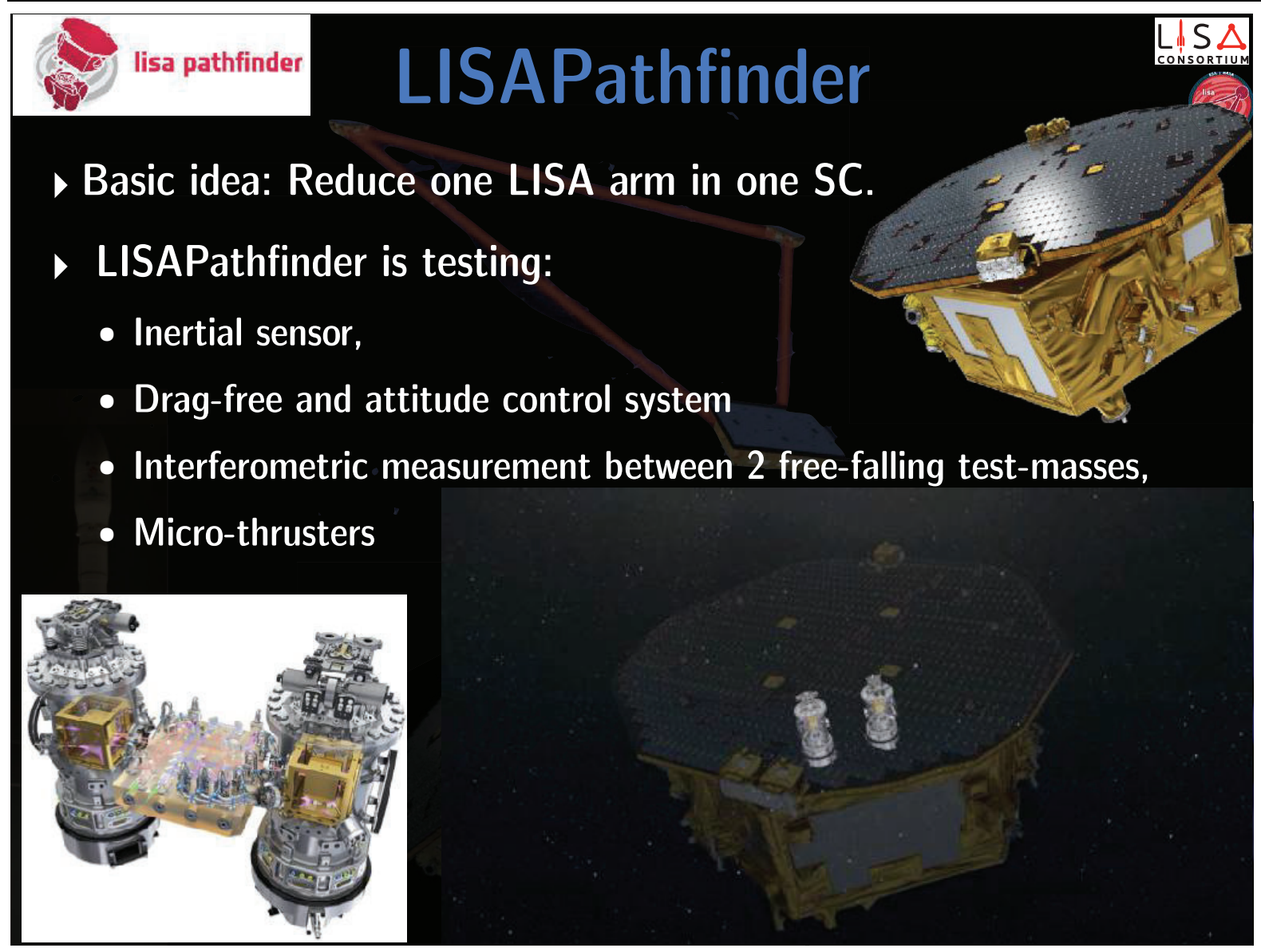

\section{LISAPathfinder timeline}




\section{LISAPathfinder timeline}

- 3/12/2015: Launch from Kourou

- 22/01/2016: arrived on final orbit \& separation of propulsion module

17/12/2015 $\rightarrow$ 01/03/2016: commissioning

$\checkmark$ 01/03/2016 $\rightarrow$ 27/06/2016: LTP operations (Europe)

> 27/06/2016 $\rightarrow$ 11/2016: DRS operations (US) + few LTP weeks

> 01/12/2016 $\rightarrow$ 31/06/2017: extension of LTP operations

18/07/2017: Last command

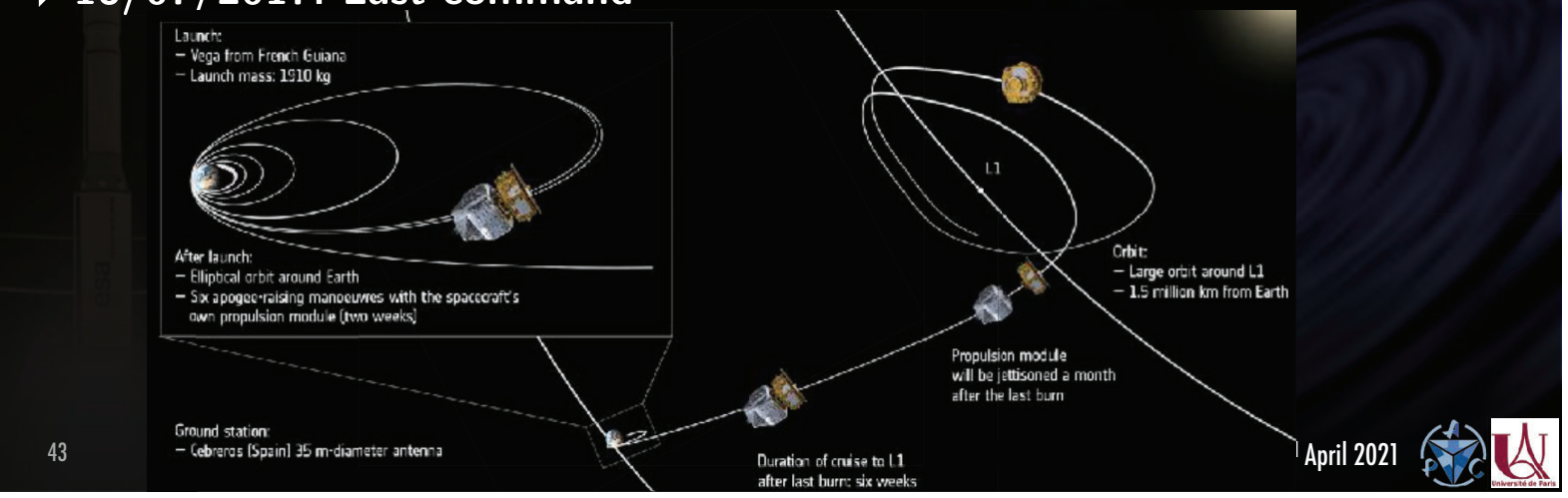

\section{The measurement - deltaG}
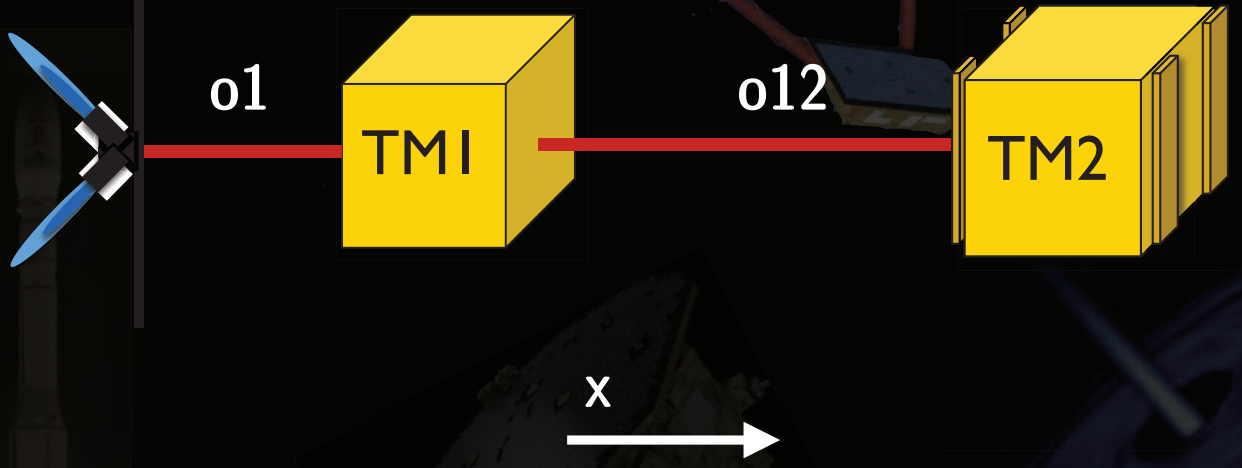

${ }_{44}$ by Joseph Martino 


\section{First results}

\section{Armano et al. PRL 116, 231101 (2016)}

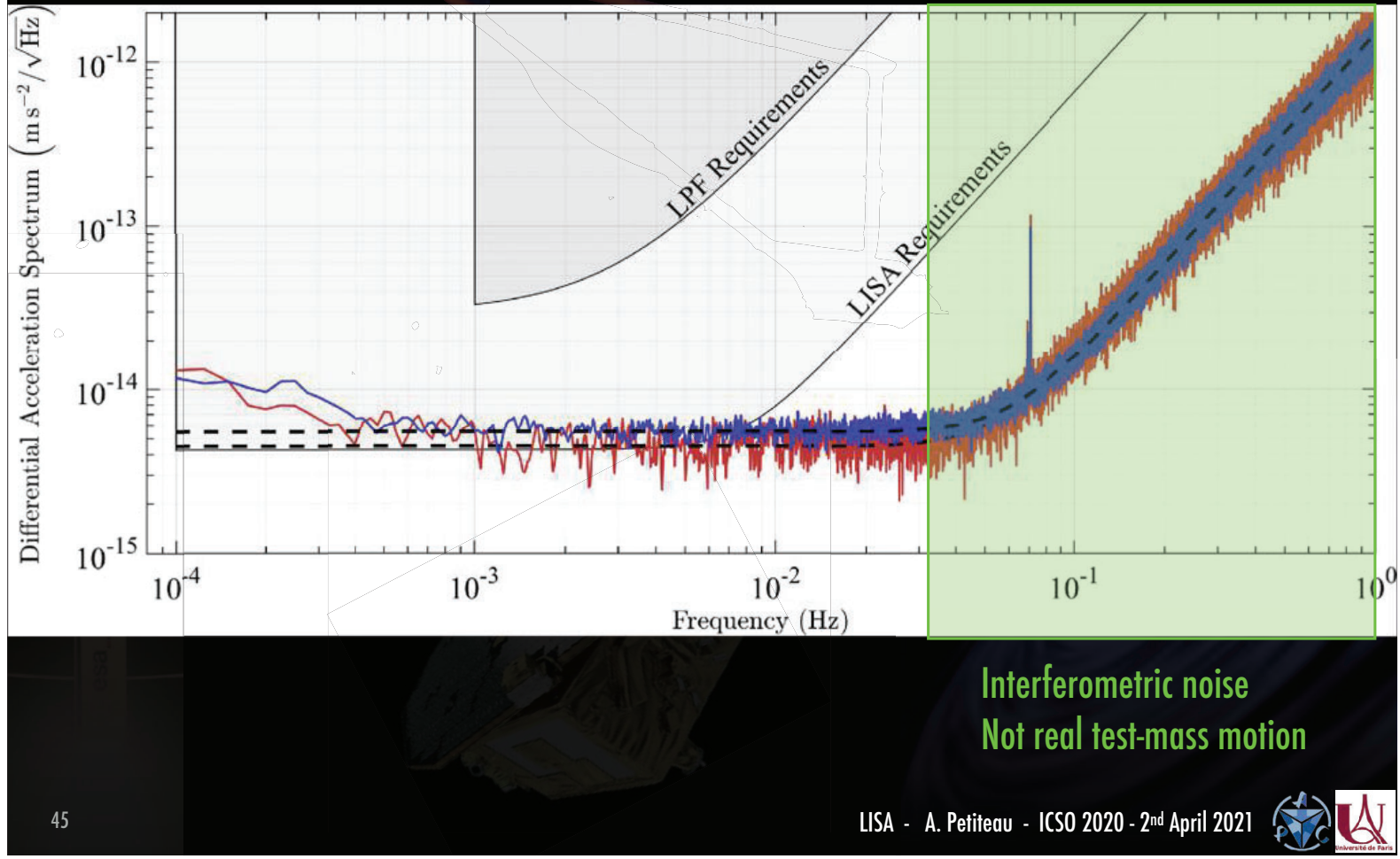

\section{High frequency limit}

\section{> Optical measurement system:}

- Interferometric precision: $30 \mathrm{fm} \cdot \mathrm{Hz}^{-1 / 2}$

- Orientation of test-masses

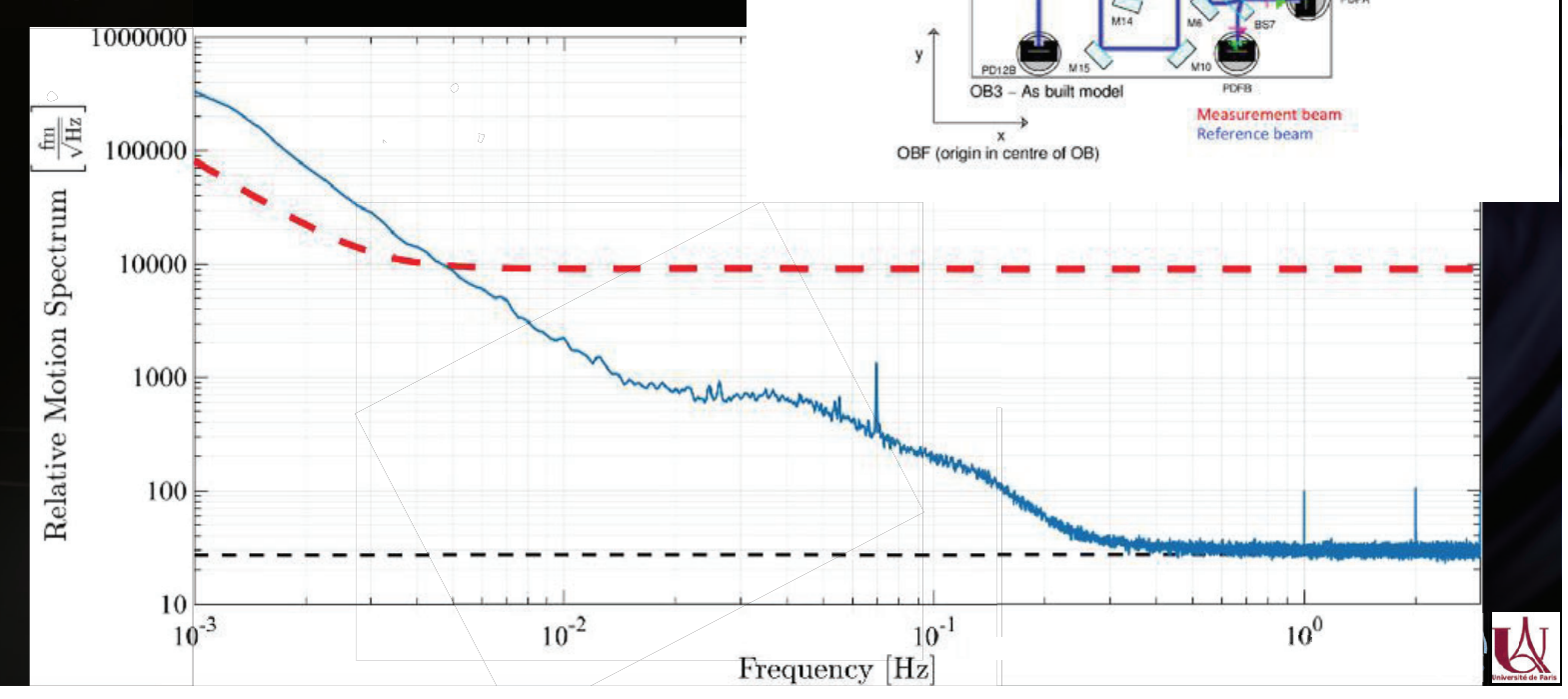




\section{First results}

M. Armano et al. PRL 116, 231101 (2016)

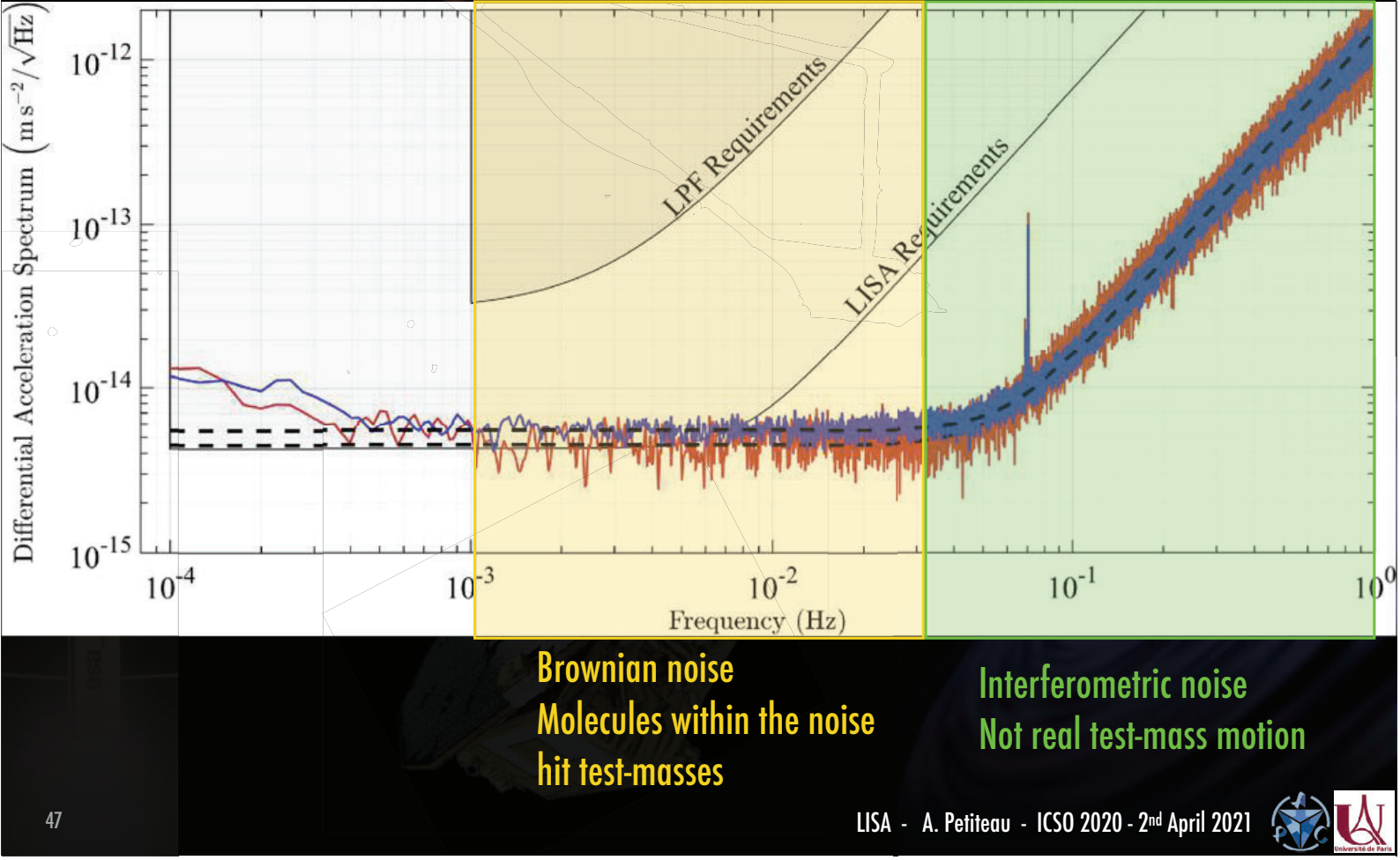

\section{Mid-frequency limit}

> Noise in 1-10 mHz:

brownian noise due to residual pressure:

- Molecules within the housing hitting the testmasses

- Possible residual outgassing

D Evolution:

- Pressure decreases with time $=>$ constant improvement

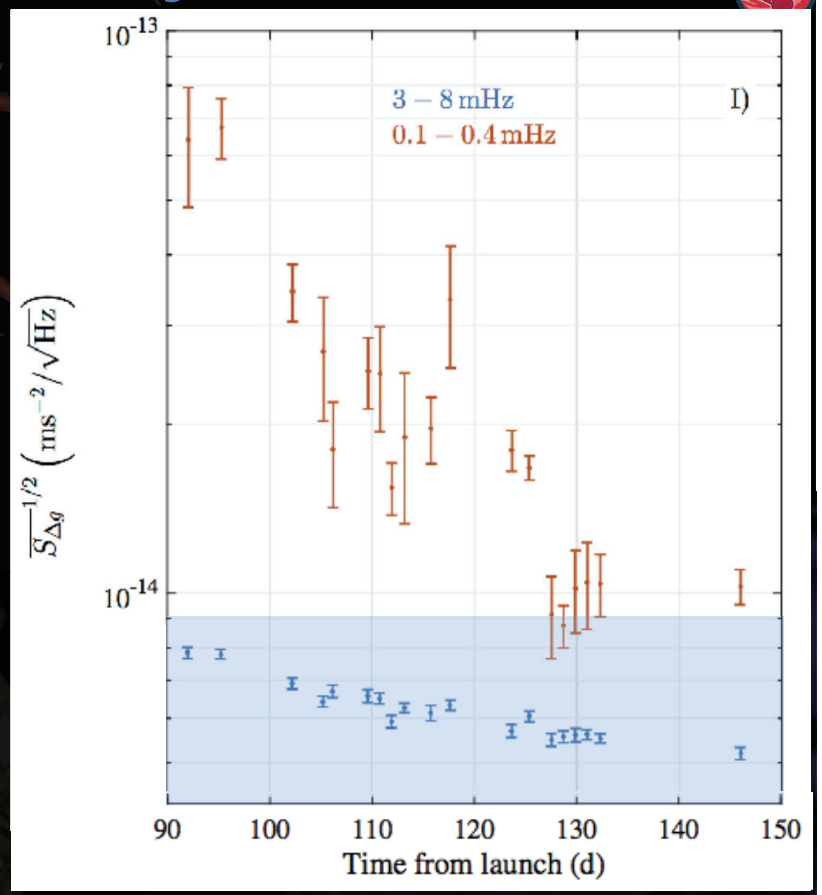

M. Armano et al. PRL 116, 231101 (2016) 


\section{First results}

M. Armano et al. PRL 116, 231101 (2016)

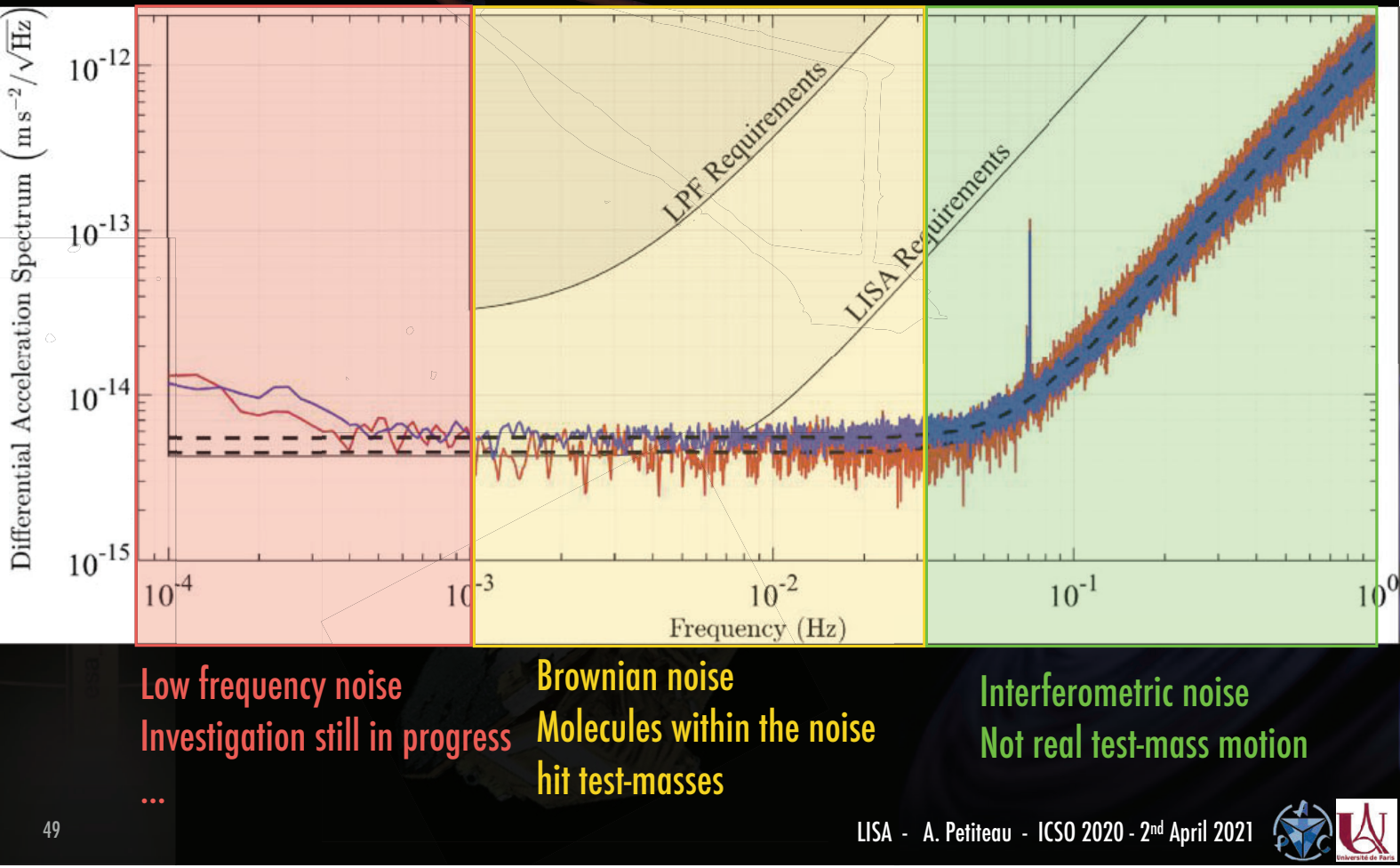

\section{Low-frequency limit}

Noise in $0.1-1 \mathrm{mHz}$ :

- $50 \%$ understood: actuation noises

- Still $50 \%$ not completely explained:

- $1 / \mathrm{f}$ slope

- Temperature? Small glitches?

- Still work in progress ...

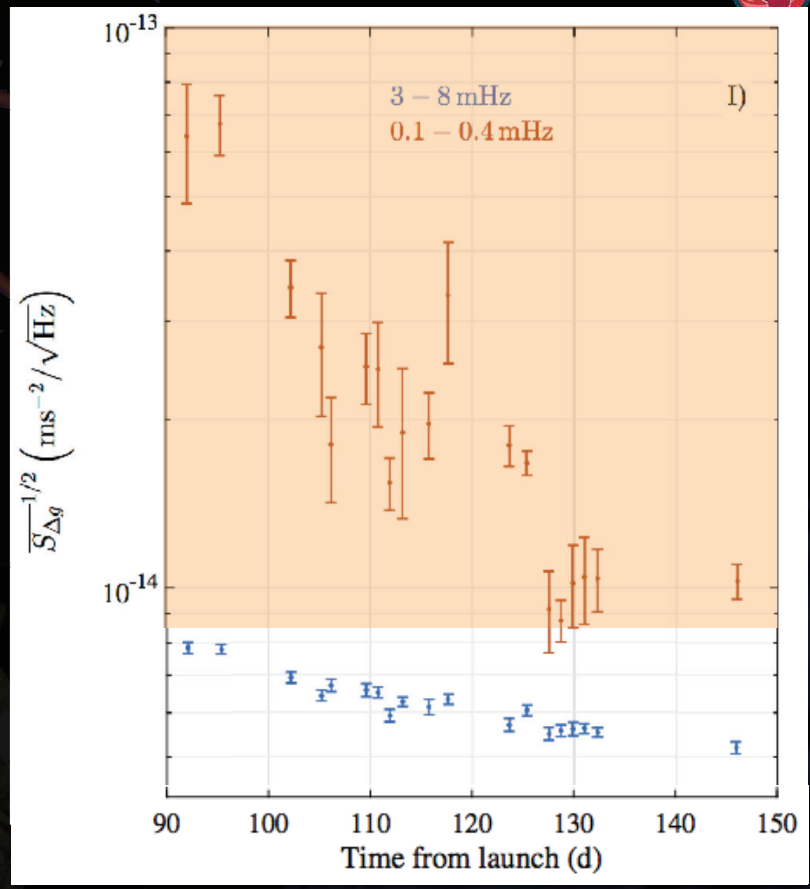

M. Armano et al. PRL 116, 231101 (2016) 


\section{LISAPathfinder final main results}

M. Armano et al. PRL 120, 061101 (2018)

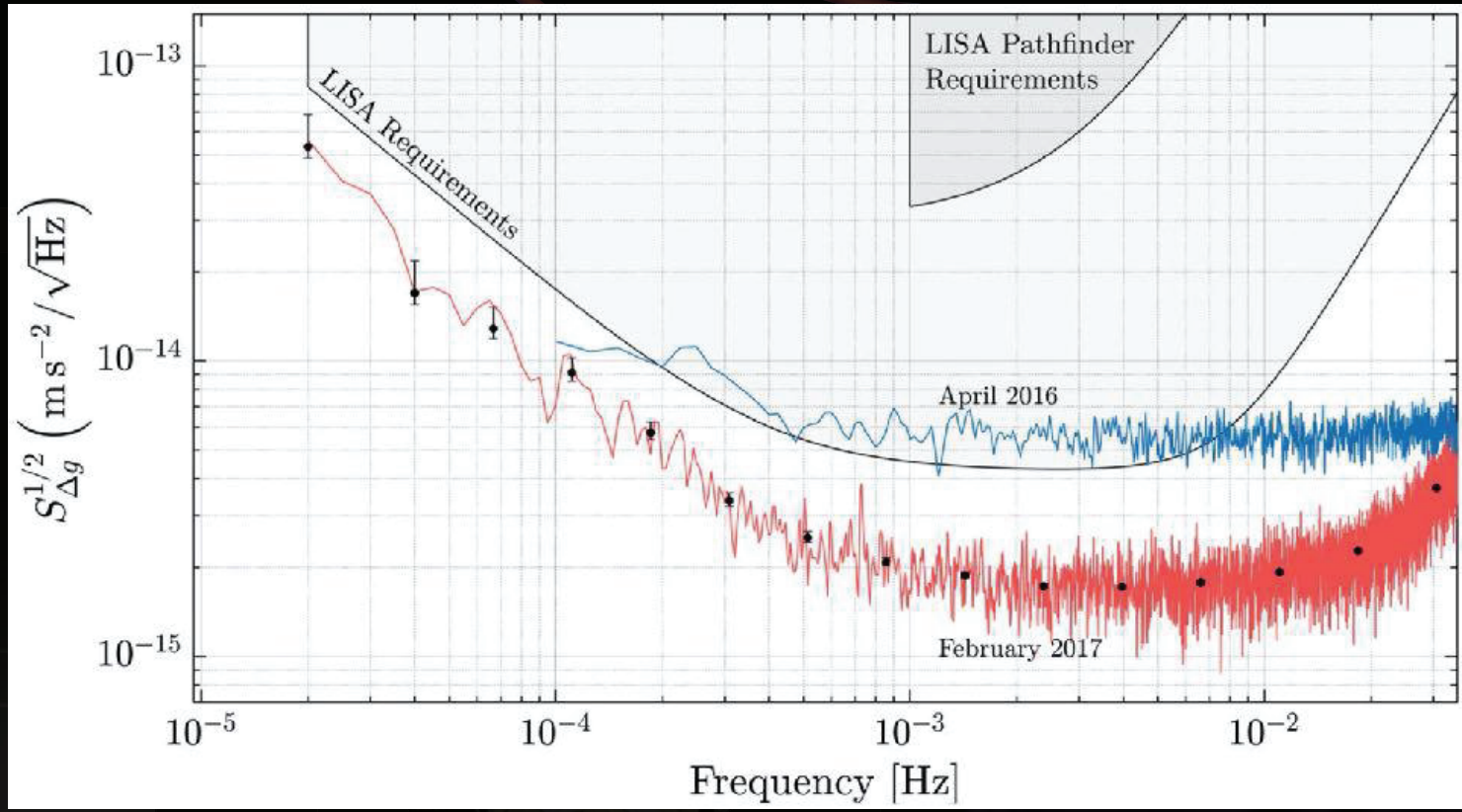

\section{LISA technology requirements}

- Free flying test mass subject to very low parasitic forces:

$\checkmark$ Drag free control of spacecraft (non-contacting) with low noise microthruster

$\checkmark$ Large gaps, heavy masses with caging mechanism

$\checkmark$ High stability electrical actuation on cross degrees of freedom

$\checkmark$ Non contacting discharging of test-masses

$\checkmark$ High thermo-mechanical stability of spacecraft

$\checkmark$ Gravitational field cancellation

- Precision interferometric, local ranging of test-mass and spacecraft:

$\checkmark$ pm resolution ranging, sub-mrad alignments

$\checkmark$ High stability monolithic optical assemblies

- Precision million $\mathrm{km}$ spacecraft to spacecraft precision ranging:

$\Rightarrow$ High accuracy laser frequency stabilisation + noise suppression with TDI

- "Tilt to length" coupling (control of alignement + ground correction)

$\Rightarrow$ Low level of stray-light

- High stability telescopes

- High accuracy phase-meter and frequency distribution

$\rightarrow$ Constellation acquisition

$\checkmark$ Precision attitude control of spacecraft

20 - 2nd April 2021 


\section{Outline}

- Introduction to gravitational waves

- Gravitational wave sources

\ Gravitational waves observatories

- LISA mission

- LISAPathfinder

- LISA status

- LISA scientific performances

Conclusion

\section{History of LISA}

1978: first study based on a rigid structure (NASA)

1980s: studies with 3 free-falling spacecrafts (US)

1993: proposal ESA/NASA: 4 spacecrafts

1996-2000: pre-phase A report

- 2000-2010: LISA and LISAPathfinder: ESA/NASA mission

- 2011: NASA stops $=>$ ESA continue: reduce mission

- 2012: selection of JUICE L1 ESA

\2013: selection of ESA L3 : « The gravitational Universe 》

- 2015-2016: success of LISAPathfinder + detection GWs

\section{Call for mission at ESA}




\section{LISA at ESA}

- 25/10/2016 : Call for mission

\13/01/2017 : submission of «LISA proposal»(LISA consortium)

- 8/3/2017 : Phase 0 mission (CDF 8/3/17 $\rightarrow$ 5/5/17)

- 20/06/2017 : LISA mission approved by SPC

- 8/3/2017 : Phase 0 payload (CDF June $\rightarrow$ November 2017)

- 2018 $\rightarrow 2021$ : phase A: payload study + competitive studies for 2 primes

\2021 $\rightarrow 2023$ : phase B1

\ 2024 : mission adoption

- During about 10 years : production: challenge (3 S/Cs with 2 MOSAs)

- 2034 : launch Ariane 6.4

- 1.5 years for transfert

- 6 - 12 months for commissioning

- 4-6 years of nominal mission ( $75 \%$ duty cycle)

- Possible extension to 10 years

GW observations!

\section{LISA Status}

- Approved ESA project with NASA as a junior partner

- Very active design and prototyping activity

- LISA Consortium:

- Key expert group for observatory formulation \& implementation

- About 1400 members with half full (direct contribution) and half associates.

- Organise LISA scientific community

- Coordination the development of data analysis and scientific analysis

- Coordinating deliverables from ESA member states 


\section{LISA Consortium}

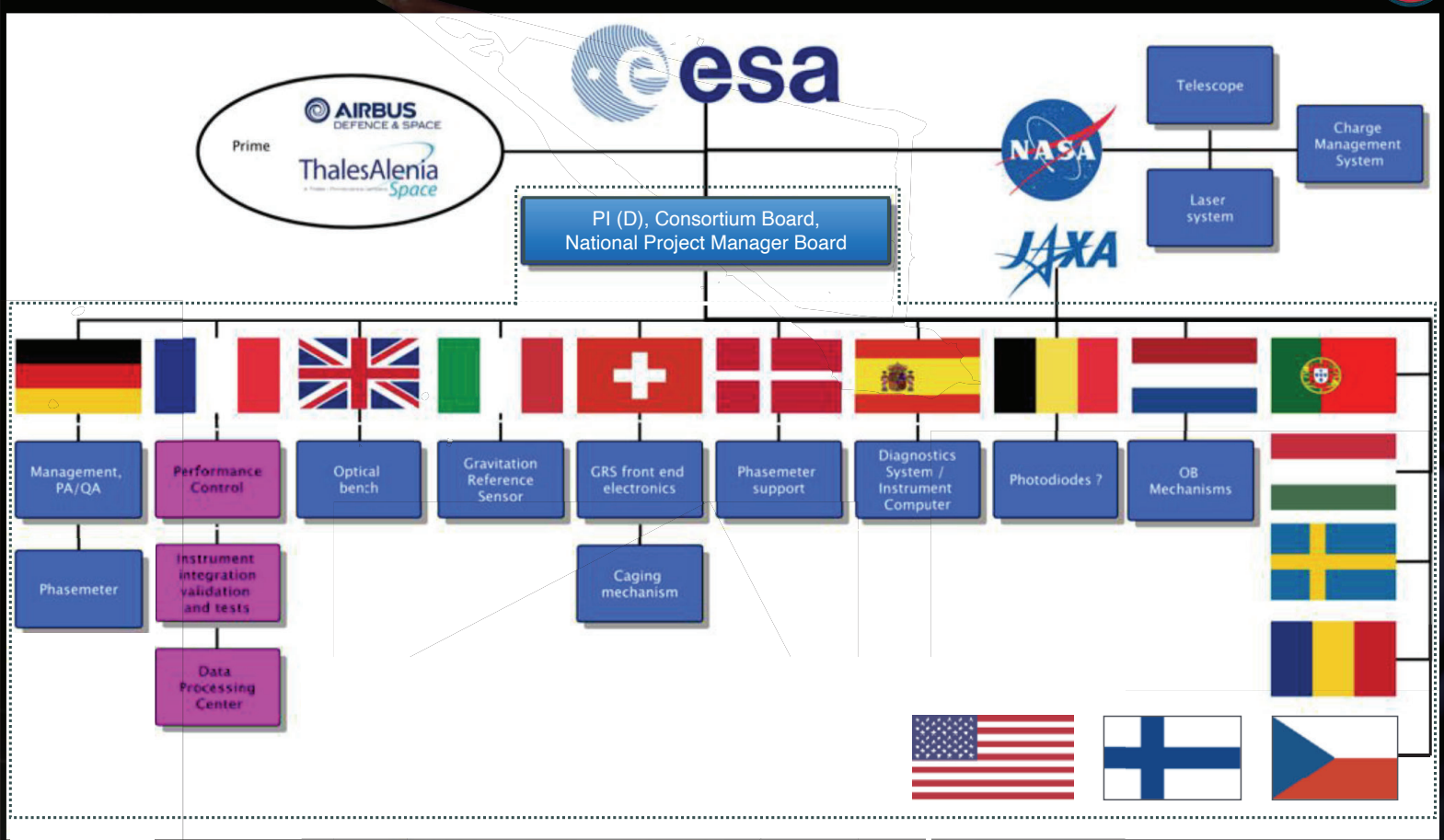

\section{Outline}

- Introduction to gravitational waves

- Gravitational wave sources

- Gravitational waves observatories

- LISA mission

- LISAPathfinder

- LISA status

- LISA scientific performances

- Conclusion 


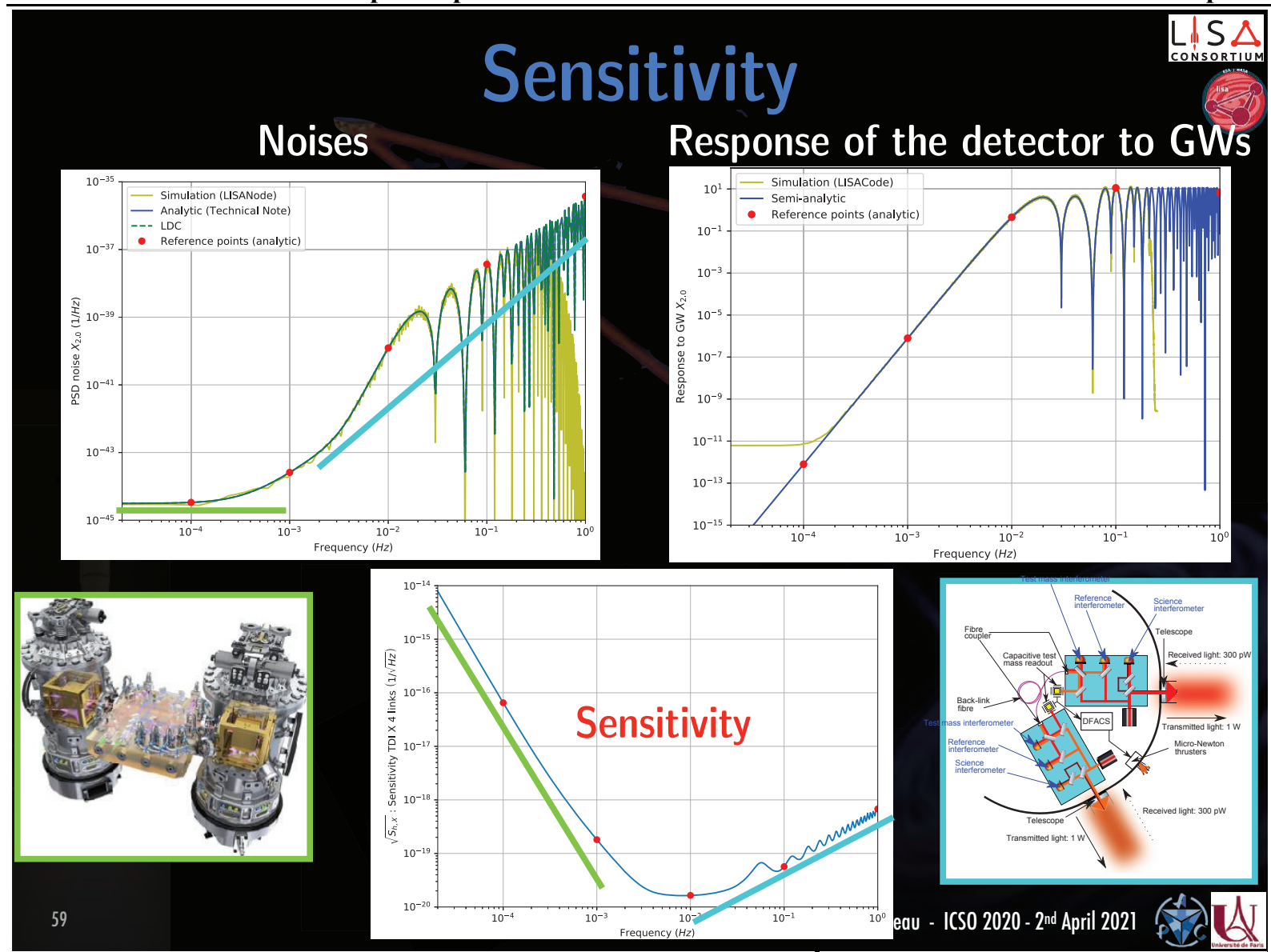

\section{Galactic binaries}

- Gravitational wave:

- quasi monochromatic

- Duration: permanent

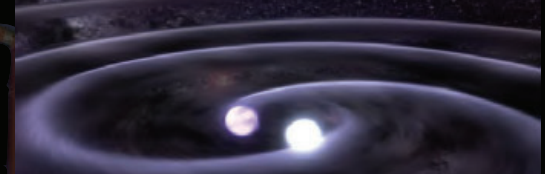

- Signal to noise ratio:

- detected sources: 7 - 1000

- confusion noise from non-detected sources

- Event rate:

- 25000 detected sources (over 30 millions sources)

- more than 10 guarantied sources (verification binaries) 


\section{Galactic binaries}

\section{GW sources}

- $6 \times 10^{7}$ galactic binaries

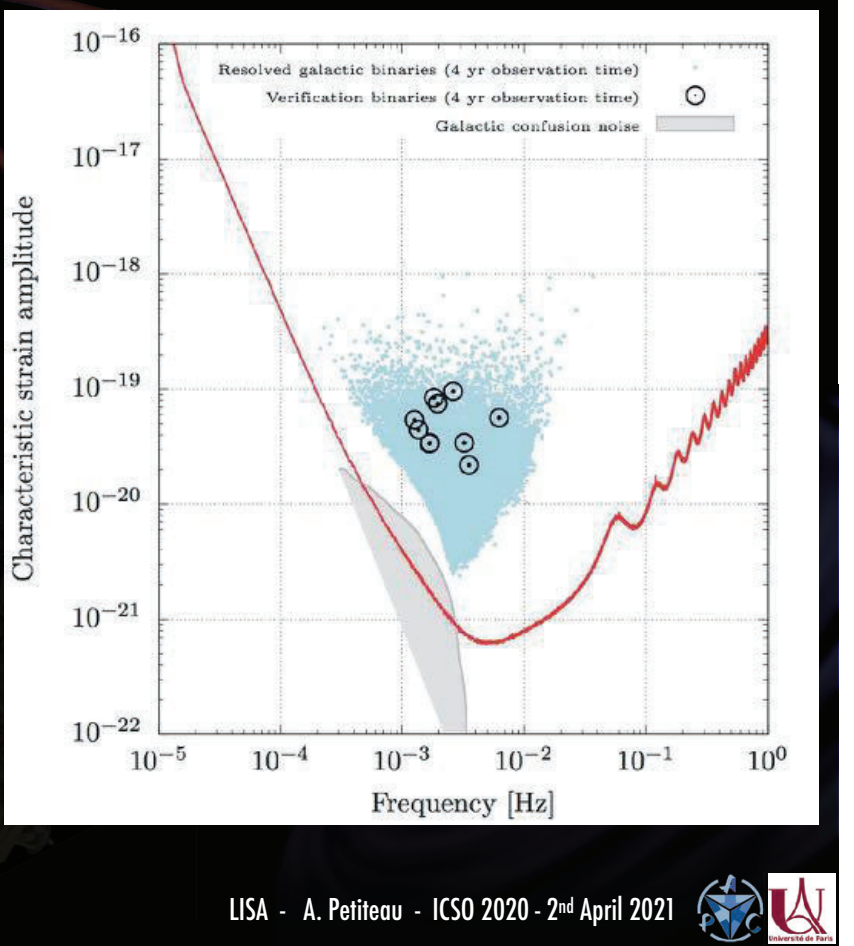

\section{Super Massive Black Hole Binaries:ss}

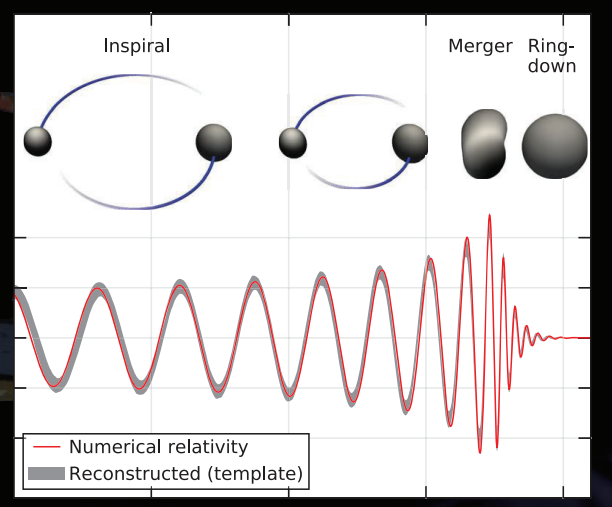

- Gravitational wave:

- Inspiral: Post-Newtonian,

- Merger: Numerical relativity,

- Ringdown: Oscillation of the resulting $\mathrm{MBH}$.

Li, $S, \Delta$

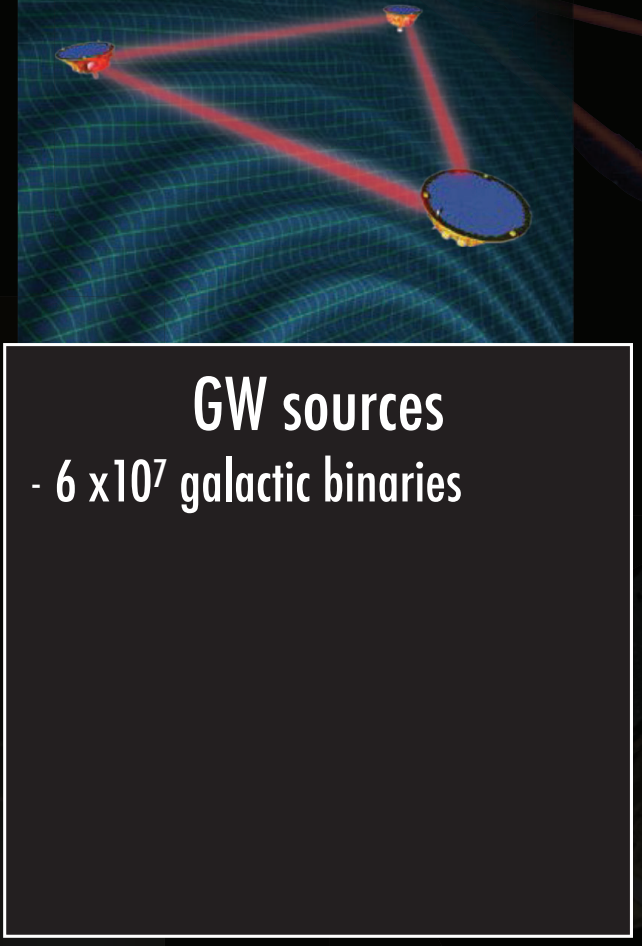

PrC 6 


\section{Super Massive Black Hole Binaries}

\section{LISA: SMBHB from $10^{4}$ à $10^{7}$ solar masses in "all" Univers}

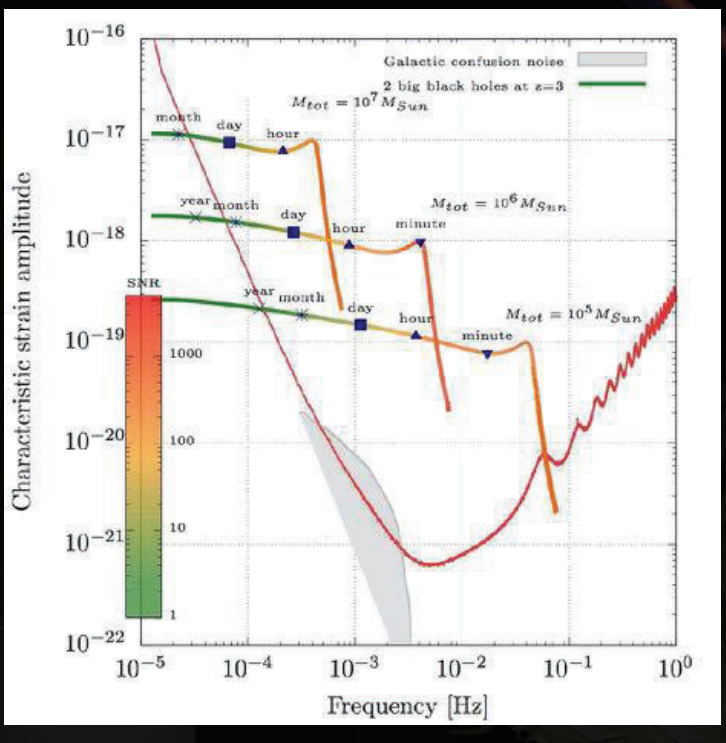

63

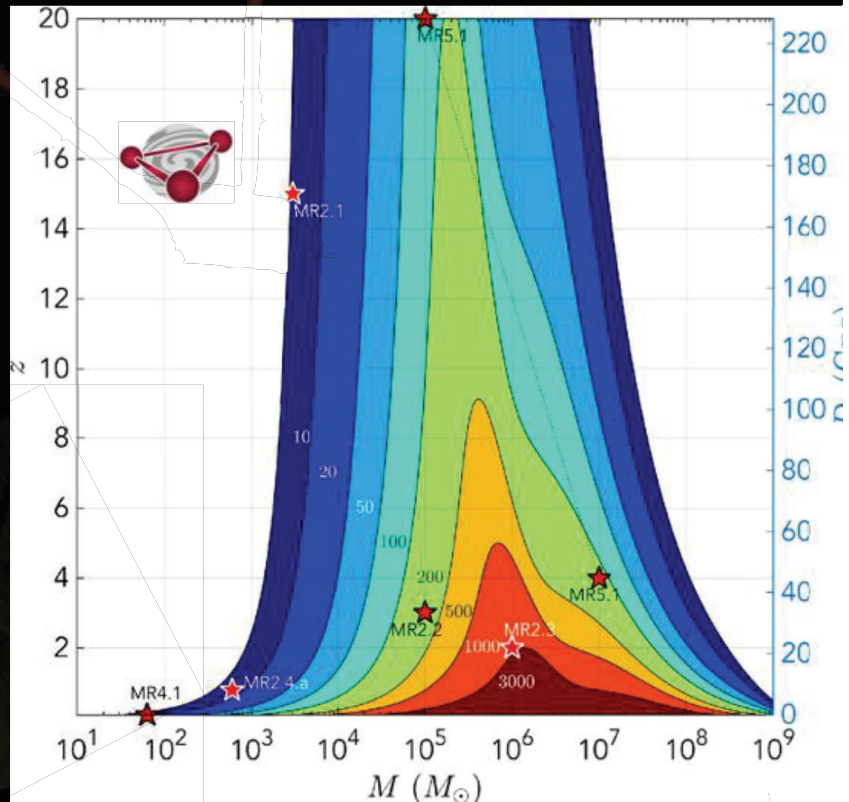

LISA - A. Petiteau - ICSO 2020 - 2nd April 2021 CAL

\section{Super Massive Black Hole Binaries}
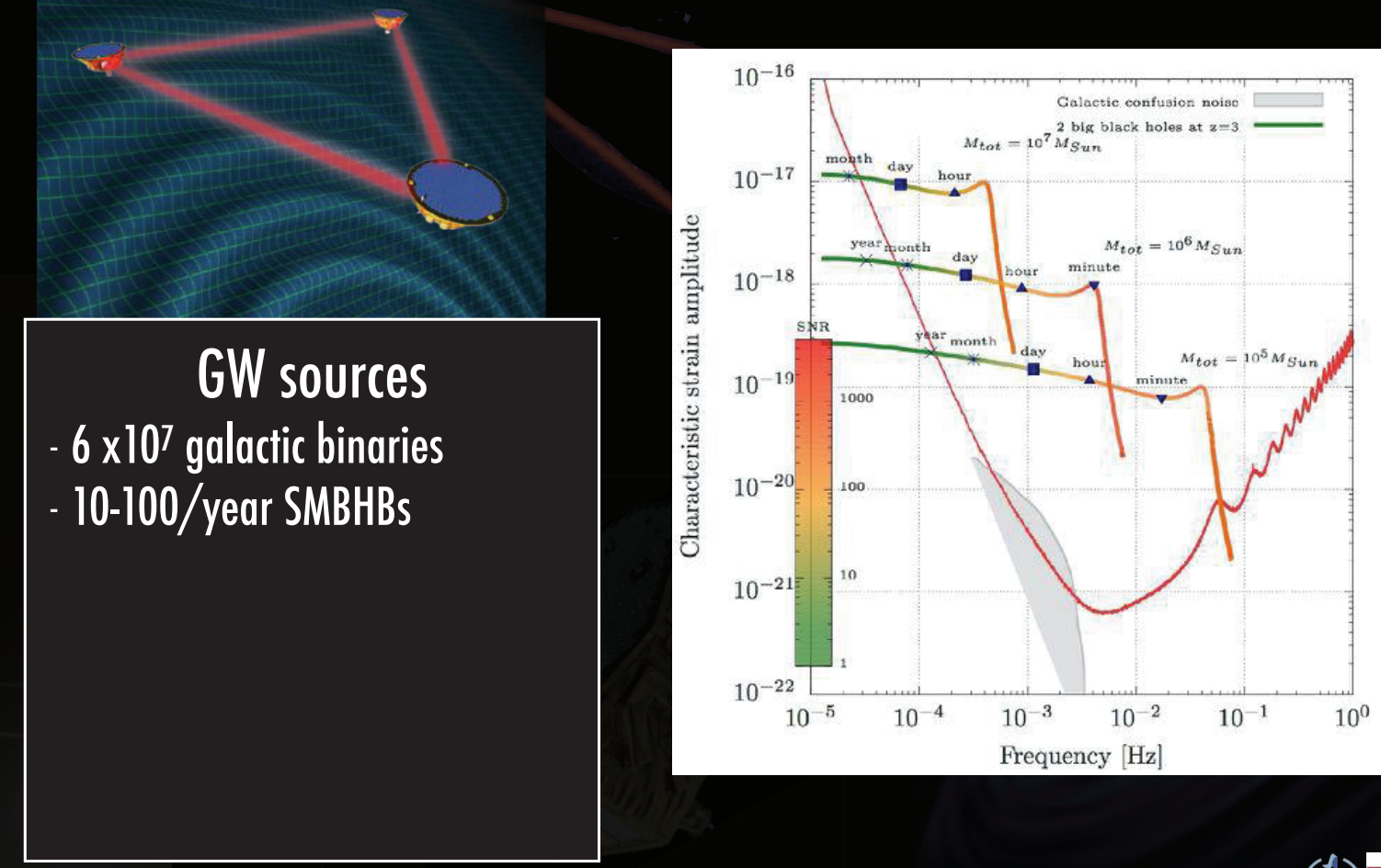


\section{EMRls}

\ Gravitational wave:

- very complex waveform

- No precise simulation at the moment

- Duration: about 1 year

- Signal to Noise Ratio: from tens to few hundreds

Dvent rate:

from few events per

year to few

hundreds

\section{EMRls}

\section{GW sources}

$.6 \times 10^{7}$ galactic binariess

- 10-100/year SMBHBs

- 10-1000/years EMRls

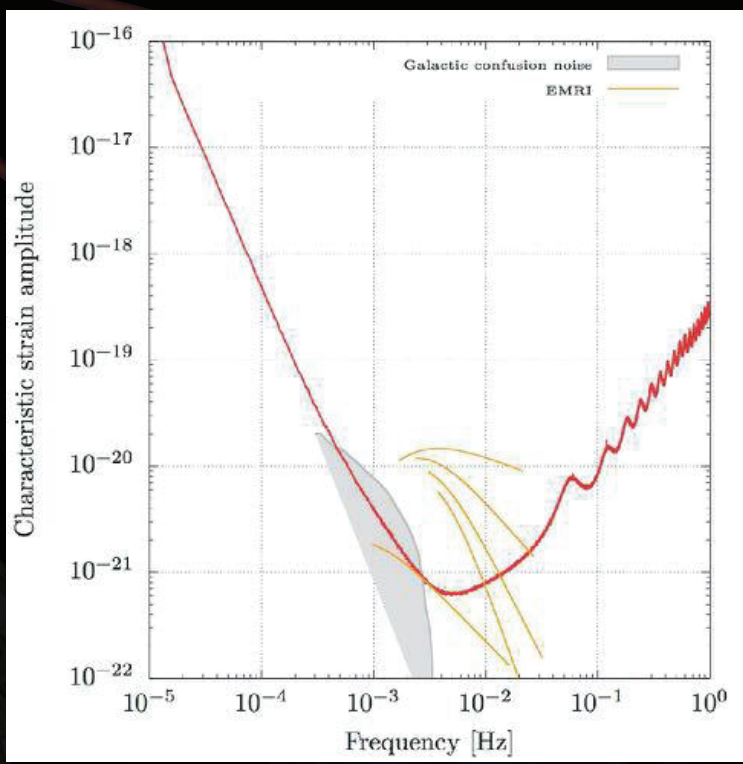




\section{Cosmological backgrounds}

- Potential detection of cosmological background from:

- First order phase transition in the very earlyUniverse
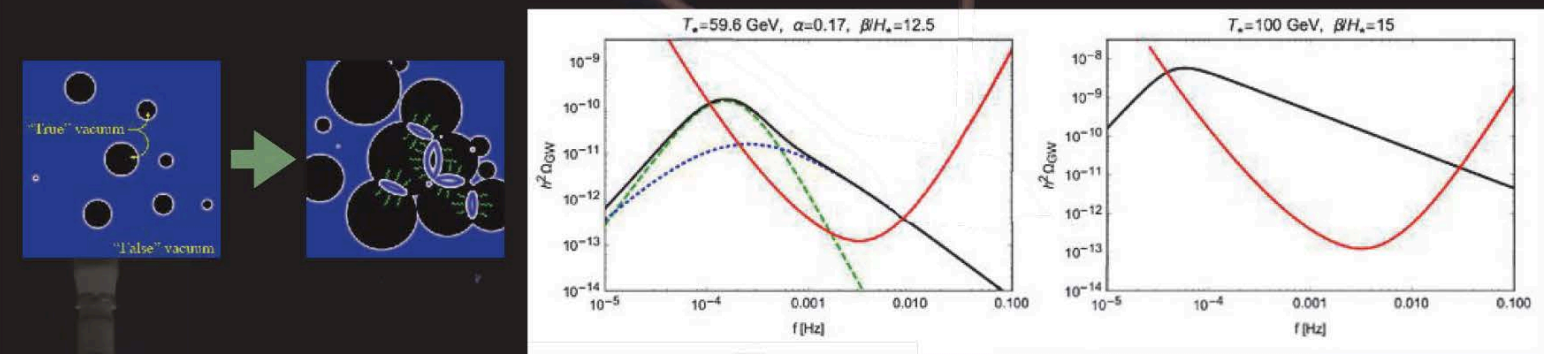

Caprini \& Figueroa 2018, CQG 35,163001

- Cosmic strings network

0
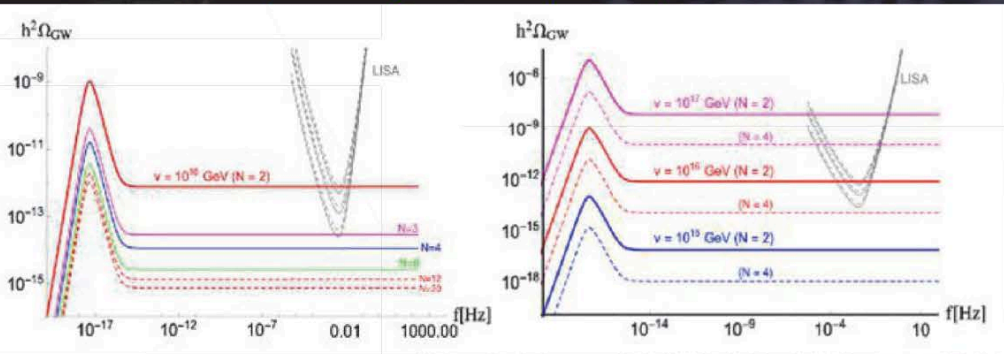

\section{GW sources}

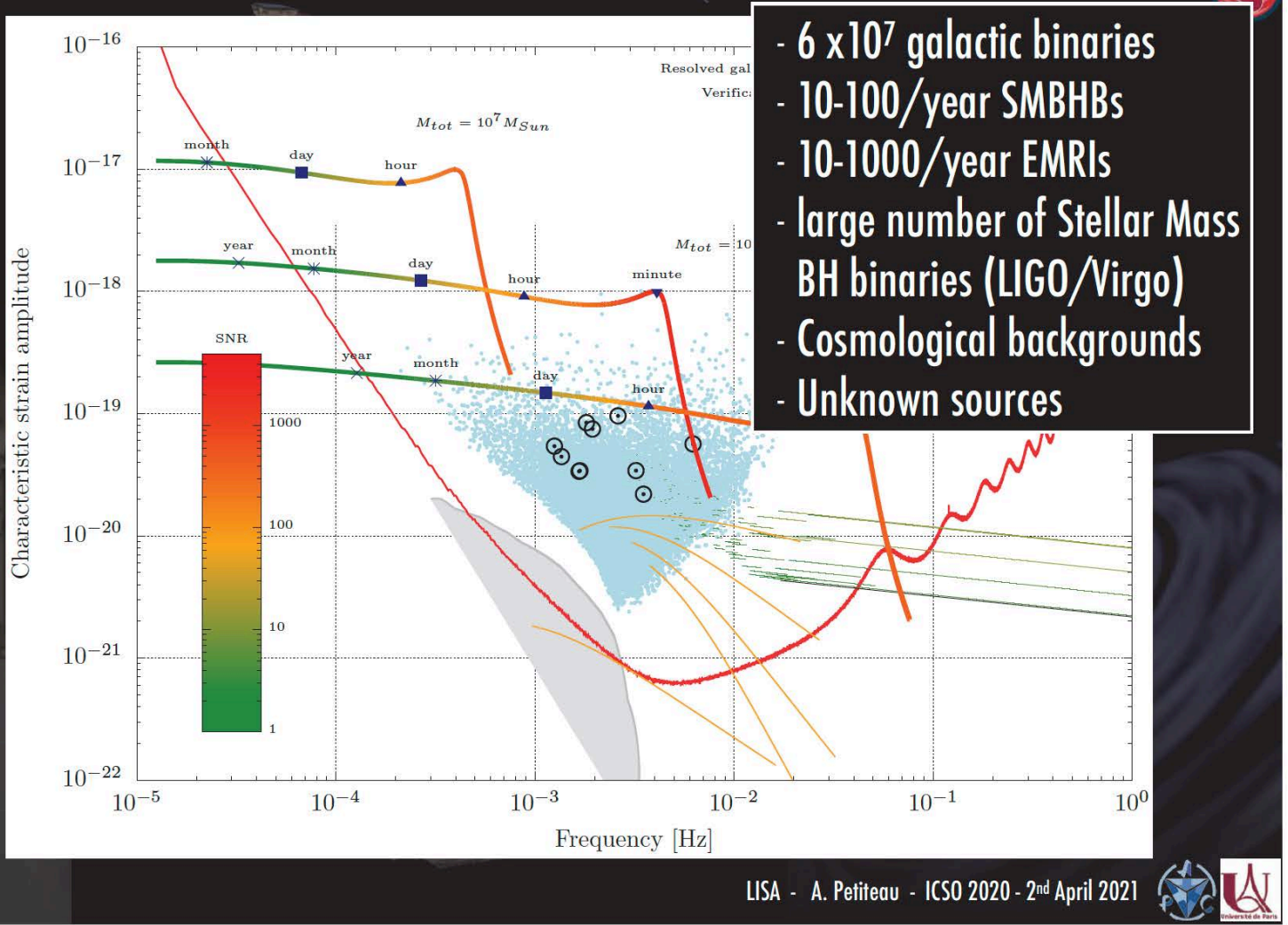




\section{LISA science objectives}

- S01: Study the formation and evolution of compact binary stars in the Milky Way Galaxy.

- S02: Trace the origin, growth and merger history of massive black holes across cosmic ages

- S03: Probe the dynamics of dense nuclear clusters using EMRIs

- S04: Understand the astrophysics of stellar origin black holes

- S05: Explore the fundamental nature of gravity and black holes

- S06: Probe the rate of expansion of the Universe

- S07: Understand stochastic GW backgrounds and their implications for the early Universe and $\mathrm{TeV}$-scale particle physics

- S08: Search for GW bursts and unforeseen sources

69

\section{Summary}

- Gravitational waves are new way to observe the Universe:

- Huge scientific potential: physic, astrophysics, cosmology, ...

- LISA: space-based interferometer

- Success of the technological demonstration with LISAPathfinder

- ESA mission with a large scientific Consortium

- In phase A for a launch 2034

- Very integrated system: the instrument includes spacecrafts and part of the ground segment

- Requires an extremely high precision in metrology

- Will observe a large number and variety of GW sources in the frequency band $10^{-5}$ to $1 \mathrm{~Hz}$ $=>$ will bring a new vision of our Universe 


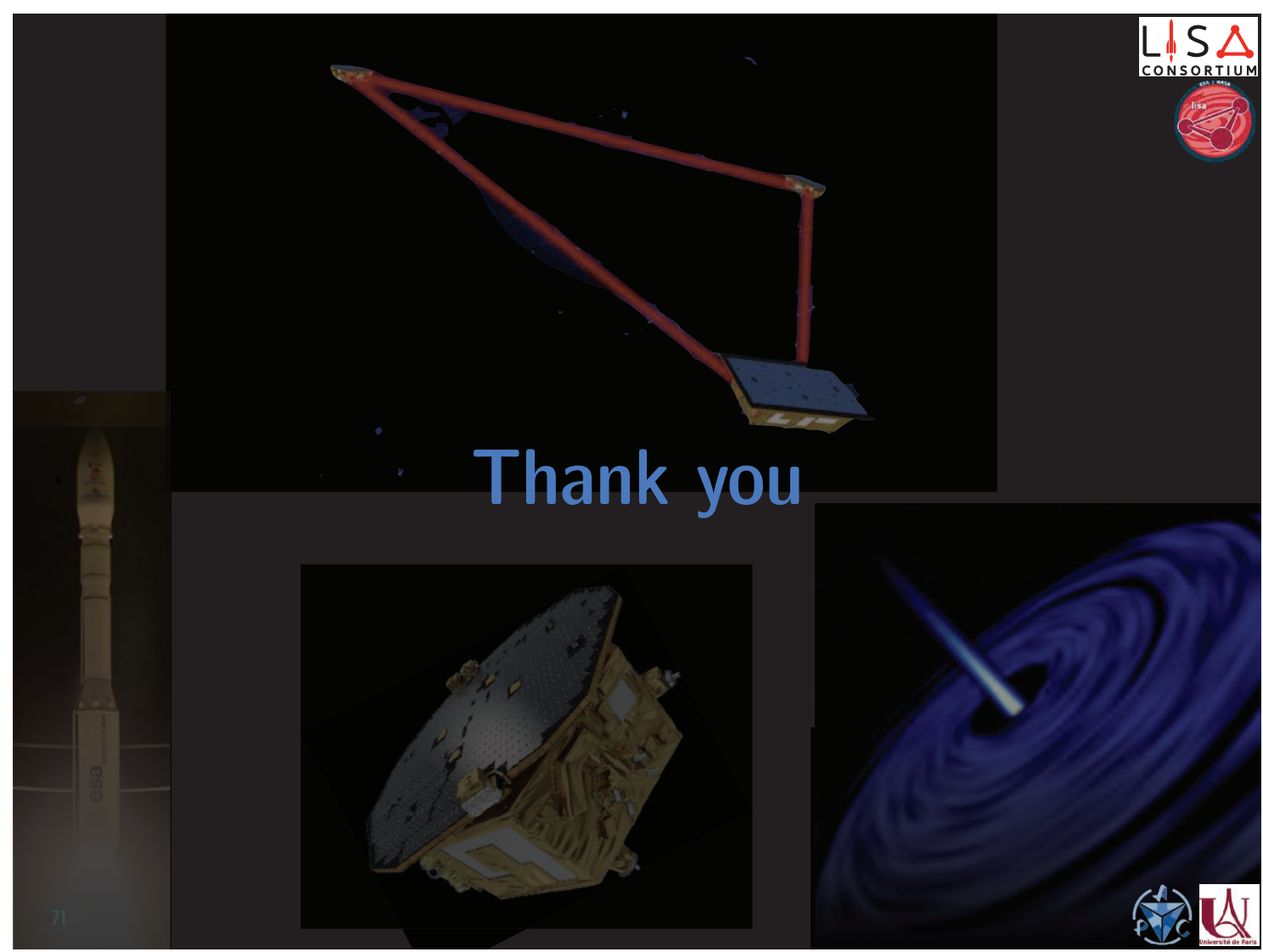

Proc. of SPIE Vol. 11852 1185205-37 